\title{
Time-Dependent Reliability Modeling and Analysis Method for Mechanics Based on Convex Process
}

\author{
Lei Wang, Xiaojun Wang, Ruixing Wang, and Xiao Chen \\ Institute of Solid Mechanics, Beihang University, Beijing 100191, China \\ Correspondence should be addressed to Xiaojun Wang; xjwang@buaa.edu.cn
}

Received 1 August 2014; Revised 30 March 2015; Accepted 30 March 2015

Academic Editor: Nenad Mladenovic

Copyright (C) 2015 Lei Wang et al. This is an open access article distributed under the Creative Commons Attribution License, which permits unrestricted use, distribution, and reproduction in any medium, provided the original work is properly cited.

\begin{abstract}
The objective of the present study is to evaluate the time-dependent reliability for dynamic mechanics with insufficient timevarying uncertainty information. In this paper, the nonprobabilistic convex process model, which contains autocorrelation and cross-correlation, is firstly employed for the quantitative assessment of the time-variant uncertainty in structural performance characteristics. By combination of the set-theory method and the regularization treatment, the time-varying properties of structural limit state are determined and a standard convex process with autocorrelation for describing the limit state is formulated. By virtue of the classical first-passage method in random process theory, a new nonprobabilistic measure index of time-dependent reliability is proposed and its solution strategy is mathematically conducted. Furthermore, the Monte-Carlo simulation method is also discussed to illustrate the feasibility and accuracy of the developed approach. Three engineering cases clearly demonstrate that the proposed method may provide a reasonable and more efficient way to estimate structural safety than Monte-Carlo simulations throughout a product life-cycle.
\end{abstract}

\section{Introduction}

Structural reliability assessment aims at computing the probability/possibility of failure occurrence for a mechanical system with reference to a specific failure criterion by accounting for uncertainties arising in the model description or the environment [1]. Essentially, the reliability measurement represents safety level in industry practice and hence the capability to efficiently perform reliability analysis is of vital importance in practical engineering applications $[2,3]$. Currently, two main categories of reliability analysis are, respectively, the time-independent (static) reliability analysis and the timedependent reliability analysis [4]. Over the past few decades, many efforts have been focused on static reliability research methodologies including probabilistic $[5,6]$, nonprobabilistic $[7,8]$, and hybrid $[9,10]$ models. Therefore, the timeindependent reliability theory has made great progress in the reliability estimate of all kinds of industrial systems and becomes the most universal theory when dealing with uncertainties.

However, in view of the comprehensive reasons of material property degeneration, varying environmental conditions, and dynamic load processes, the uncertain structures in practical engineering still exhibit a distinct time-varying effect [11]. Although the approaches based on static reliability theory have been widely used to estimate the structural safety recently, how to ensure high reliability level during a product life-cycle is still a big challenge in engineering applications. To tackle the time-dependency issues, Lots of research results have been published in time-dependent reliability analysis in recent years. They include the extreme value distribution method $[12,13]$, the first-passage method $[1,14]$, the Markov chain method [15], and the Monte-Carlo simulation method [16]. Among them, the first-passage method based on Rice's formula [17] is recognized as the most popular method in current literature of reliability analysis research. It concentrates on the first time when the performance function exceeds the upper bound or falls below the lower bound of the given safety threshold, which is commonly by virtue of the calculation of the "outcrossing rate" to quantitatively evaluate the probability of failure. Since the development of Rice's formula, amounts of improvements have been made. For example, Vanmarcke proposed a commonly used improved formula, accounting for the dependence between the crossing events and the time that the process spends above the barrier in application to normal stationary random process model 
[18]; Madsen and Krenk developed an integral equation method for solving the first-passage problems [19]; a timedependent reliability analysis method with joint upcrossing rates, inspired by [19], was further developed by $\mathrm{Hu}$ and $\mathrm{Du}$ [20] for more general cases of the limit-state functions that involve time, random variables, and stochastic processes; by combination of the ideas of outcrossing and system reliability, Wang et al. [13] presented an improved subset simulation with splitting approach by partitioning the original high dimensional random process into a series of correlated, short duration, low dimensional random processes; several improved formulations for calculating the outcrossing rate based on the Poisson assumption were, respectively, extended by Schall et al. [21], Engelund et al. [22], Streicher and Rackwitz [23], and so forth. Recently, additional correlational researches have been also suggested by $[24,25]$.

Compared to static reliability analysis, the research on time-dependent reliability is still in its preliminary stage, and the following features contribute to the sources of difficulty: (1) from the analytical point of view, very few models or analysis methods have been presented in the past for the evaluation of time-dependent reliability considering the general case of non-Gaussianity, nonstationarity, and nonlinear dependency; (2) from the perspective of simulation, extremely high computational cost is needed to guarantee reasonable numerical results of time-dependent reliability, which greatly restrict the application in complex structural systems. In recent developments, the work of Sudret et al. $[1,26]$, where an analytical outcrossing rate utilizing the PHI2 method is derived, may partly overcome the abovementioned insufficiencies.

As the literature survey reveals, most of the existing studies focus on the random process models when performing the time-dependent reliability analysis while ignoring the existence of nonprobabilistic time-varying uncertainties. Furthermore, the assessment of the time-dependent reliability by random process theory must require knowledge of probabilistic descriptions for all time-varying uncertainties, which are typically determined by sufficient experimental samples. Unfortunately, for practical engineering problems, experimental samples are not always available or re sometimes very expensive to obtain so that one cannot directly establish the precise analytical model. Giving subjective assumptions for description of the uncertainty characteristics is likely to bring about a serious error of the time-dependent reliability results. In view of the above reasons, it is quite necessary and urgent to carry out time-dependent reliability research on nonprobabilistic modeling methods. Jiang et al. [27] proposed a new theory for time-varying uncertainty, namely, the "non-probabilistic convex process model" to tackle the problems of safety estimate when lacking relevant uncertainty information. However, the solution of the timedependent reliability must rely on the Monte-Carlo simulations rather than constructing an analytical model/index. Additionally, the work in [27] mainly concentrated on the case of stationary convex process; the more common case of non-stationary process was not discussed.

Generally speaking, compared with static reliability analysis, fewer studies on time-dependent reliability analysis are performed at present and its theoretical foundation is still relatively immature. In this paper, inspired by the work in $[26,27]$, we develop a new time-dependent reliability analysis approach based on the nonprobabilistic convex process model, in which a new measure index of time-dependent reliability is established and its solution procedure is deduced mathematically. The presented approach can effectively deal with the more general case containing nonstationarity and nonlinear dependency that is of particular importance in solving practical engineering problems.

The paper is organized as follows. Section 2 provides a brief review of general concept of nonprobabilistic convex process model and its relevant mathematical basis. In Section 3, combined with the foregoing convex process model and the set-theory method, the time-varying uncertainty of structural limit state is quantified. Section 4 details the presented time-dependent reliability method, and then we introduce the Monte-Carlo simulations in Section 5. The proposed methodology is demonstrated with three case studies in Section 6 and then followed by conclusions in Section 7.

\section{Notation and Classification of Nonprobabilistic Convex Process Model}

Enlightened by [27], the nonprobabilistic convex process model is introduced in this section to quantify the timevarying uncertain parameters in dynamic mechanics when facing the case of limited sample information. Here, the uncertainty of structural parameters at any time is depicted with a bounded closed interval, and the correlation between variables in different instant time points is reflected by defining a corresponding correlation function. If we discretize the contiguous convex process into a time series, the feasible domain of all interval variables belongs to a convex set. For details, see the following definitions.

Definition 1. Consider a convex process $X(t) ; \overline{X(t)}$ and $X(t)$ describe the upper and lower bound functions of $X(t)$, and hence the mean value function $X^{c}(t)$ and the radius function $X^{r}(t)$ are, respectively, given by

$$
\begin{aligned}
X^{c}(t) & =\frac{\overline{X(t)}+\underline{X(t)}}{2}, \\
X^{r}(t) & =\frac{\overline{X(t)}-\underline{X(t)}}{2} .
\end{aligned}
$$

For convenience, we also define the variance function $D_{X}(t)$ as

$$
D_{X}(t)=\left(X^{r}(t)\right)^{2}=\left(\frac{\overline{X(t)}-\underline{X(t)}}{2}\right)^{2}
$$

It is apparently indicated that the properties of uncertain variables at any time can be quantified mathematically from 


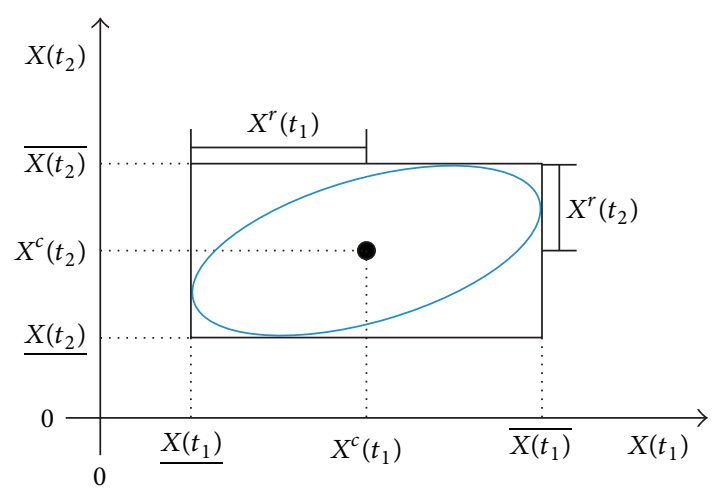

(a)

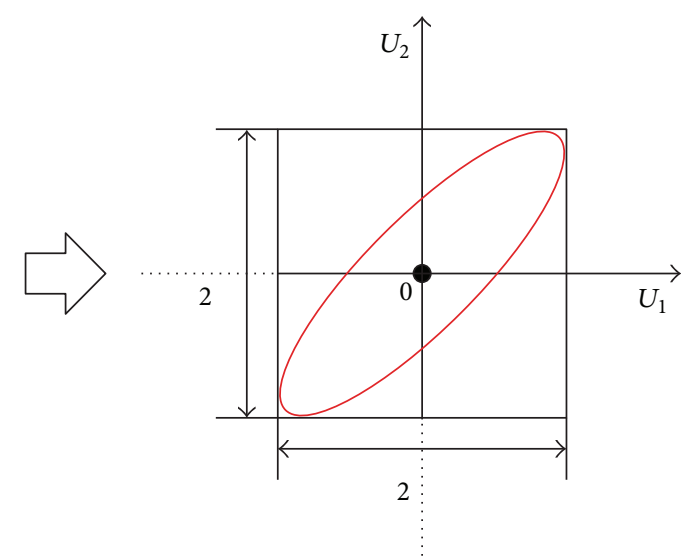

(b)

FIGURE 1: Quantitative models between interval variables at any two times in the convex process: (a) the original model; (b) the normalized model.

the above equations. The correlation between any two variables in different time points is determined by the autocovariance function or the correlation coefficient function as the following statements.

Suppose that $X\left(t_{1}\right)$ and $X\left(t_{2}\right)$ are two interval variables originating from the convex process $X(t)$ at times $t_{1}$ and $t_{2}$. In view of the existence of the correlation, a rotary ellipse model is constructed to restrict the value range of $X\left(t_{1}\right)$ and $X\left(t_{2}\right)$, as shown in Figure 1(a). By regularization treatment (from $x$ space to $u$ space), the standard model is then formed, as illustrated in Figure 1(b).

Definition 2. If $X(t)$ is a convex process, for any times $t_{1}$ and $t_{2}$, the autocovariance function of interval variables $X\left(t_{1}\right)$ and $X\left(t_{2}\right)$ is defined as

$$
\begin{aligned}
\operatorname{Cov}_{X}\left(t_{1}, t_{2}\right)= & \operatorname{Cov}\left(U_{1}, U_{2}\right) \cdot X^{r}\left(t_{1}\right) \cdot X^{r}\left(t_{2}\right) \\
= & \left(r_{m}^{2}-1\right) \cdot X^{r}\left(t_{1}\right) \cdot X^{r}\left(t_{2}\right), \\
& 0 \leq r_{m} \leq \sqrt{2},
\end{aligned}
$$

where $r_{m}$ either stands for the major axis $r_{1}$ of the ellipse in $u$ space if the slope of the major axis is positive or represents the minor axis $r_{2}$ if the slope of the major axis is negative. For details, see Figure 2.

Definition 3. With regard to the convex process $X(t)$, the autocorrelation coefficient function of $X\left(t_{1}\right)$ and $X\left(t_{2}\right)$ is expressed as

$$
\begin{aligned}
\rho_{X}\left(t_{1}, t_{2}\right) & =\frac{\operatorname{Cov}_{X}\left(t_{1}, t_{2}\right)}{\sqrt{D_{X}\left(t_{1}\right)} \cdot \sqrt{D_{X}\left(t_{2}\right)}}=\frac{\operatorname{Cov}\left(U_{1}, U_{2}\right)}{\sqrt{D_{U_{1}}} \cdot \sqrt{D_{U_{2}}}} \\
& =\rho_{U_{1} U_{2}}=r_{m}^{2}-1,
\end{aligned}
$$

where $D_{U_{1}}$ and $D_{U_{2}}$ denote the variance functions of the standard interval variables $U_{1}$ and $U_{2}\left(D_{U_{1}}=D_{U_{2}}=\right.$ 1). $\rho_{X}\left(t_{1}, t_{2}\right)$ is a dimensionless quantity and its magnitude represents the linear correlation of $X\left(t_{1}\right)$ and $X\left(t_{2}\right)$. It is obvious that $\left|\rho_{X}\left(t_{1}, t_{2}\right)\right| \leq 1$ and $\rho_{X}(t, t)=1$.

Several specific ellipse models with different values of $\rho_{X}\left(t_{1}, t_{2}\right)$ are clearly illustrated in Figure 3 . Among all the ellipses, the one with $\rho_{X}\left(t_{1}, t_{2}\right)=0$ has a maximal area, which implies the largest scattering degree and hence a minimum correlativity of variables $X\left(t_{1}\right)$ and $X\left(t_{2}\right)$. Moreover, a larger $\left|\rho_{X}\left(t_{1}, t_{2}\right)\right|$ means a stronger linear correlation between $X\left(t_{1}\right)$ and $X\left(t_{2}\right)$. Once $\left|\rho_{X}\left(t_{1}, t_{2}\right)\right|=1$, the ellipse model will be replaced by a straight line that indicates a complete linearity.

As mentioned above, the characteristics of one timevarying uncertain parameter can be commendably embodied. However, with respect to complicated structures, more common case is that we must solve the problem of multidimensional time-varying uncertainty. The cross-correlation between two convex processes should be considered as well.

Definition 4. Suppose two convex processes $X(t)$ and $Y(t)$, for any times $t_{1}$ and $t_{2}$; the cross-correlation coefficient function of $X\left(t_{1}\right)$ and $Y\left(t_{2}\right)$ is established as

$$
\begin{aligned}
\rho_{X Y}\left(t_{1}, t_{2}\right)=\frac{\operatorname{Cov}_{X Y}\left(t_{1}, t_{2}\right)}{\sqrt{D_{X}\left(t_{1}\right)} \cdot \sqrt{D_{Y}\left(t_{2}\right)}}=r_{m^{*}}^{2}-1, & \\
& 0 \leq r_{m^{*}} \leq \sqrt{2},
\end{aligned}
$$

where $\operatorname{Cov}_{X Y}\left(t_{1}, t_{2}\right)$ means the cross-covariance function and $r_{m^{*}}$ represents the major/minor axis of the ellipse in $u$ space derived from $X\left(t_{1}\right)$ and $Y\left(t_{2}\right)$ (similarly to the definition in (3)).

According to the above definitions, the characteristic parameters of nonprobabilistic convex process model are available. In the next section, associated with the set-theory method, the convex process model utilized to describe the time-varying uncertainty of structural limit state is established. 


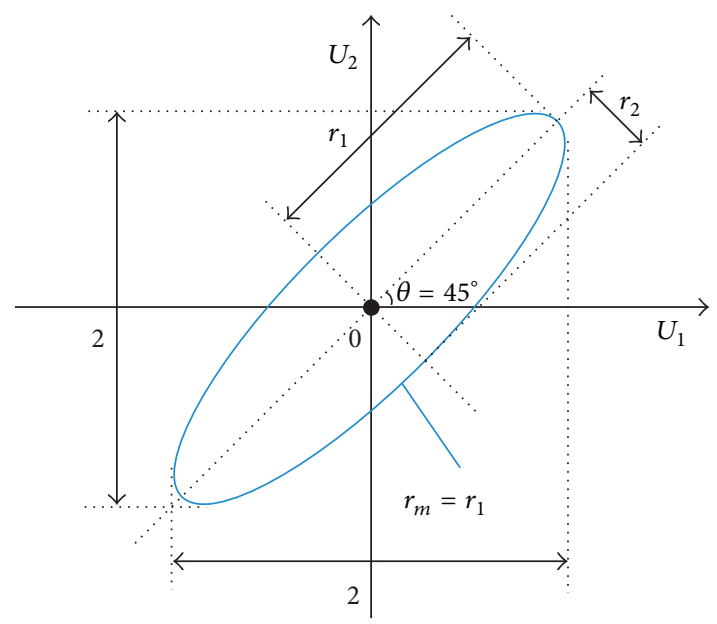

(a)

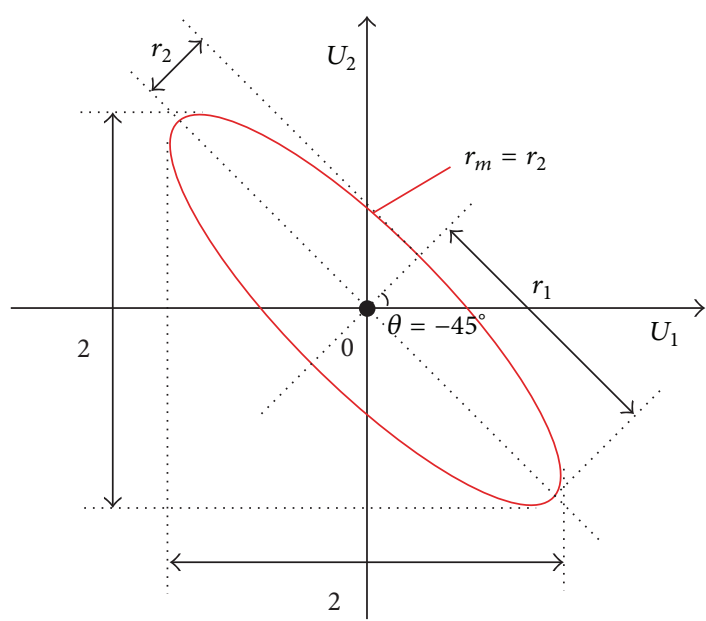

(b)

Figure 2: Definitions of autocovariance function between interval variables at any two times in the convex process: (a) the positive autocorrelation; (b) the negative autocorrelation.
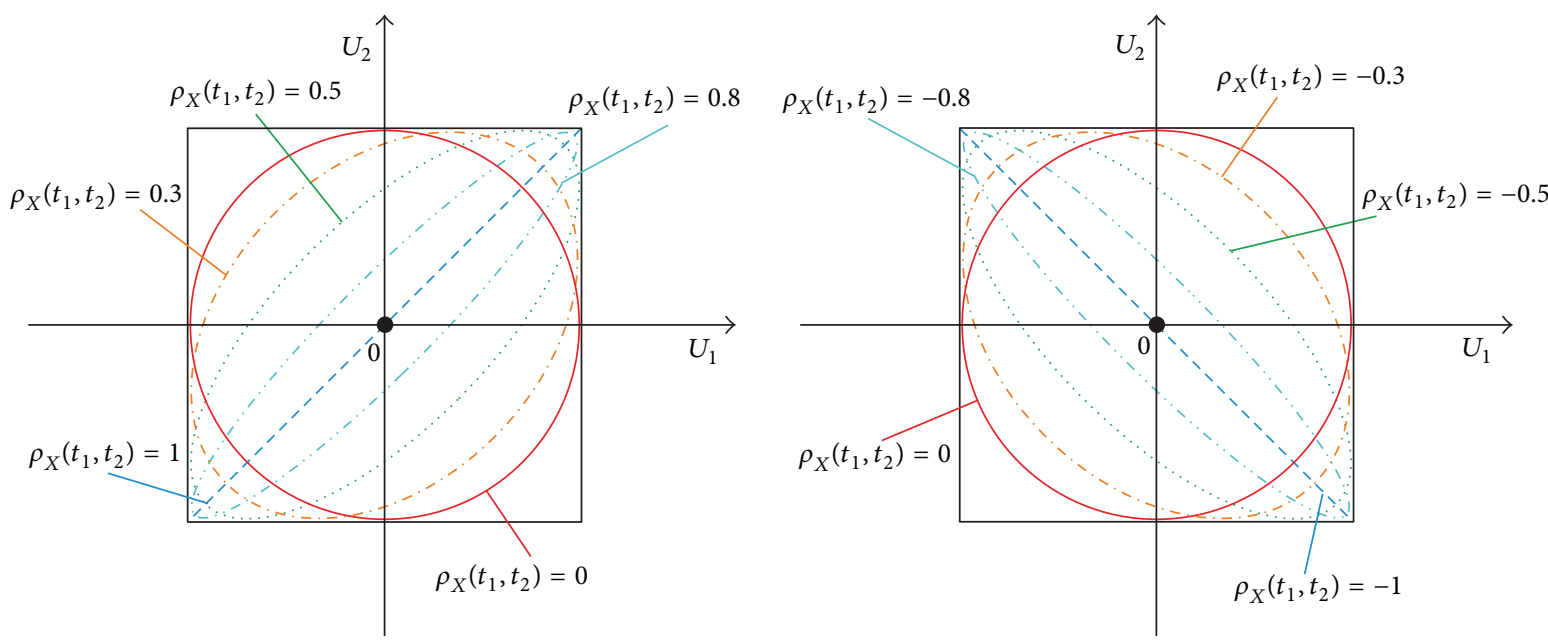

FIGURE 3: Typical geometric shapes of ellipses for different correlation coefficients.

\section{Time-Varying Uncertainty Quantification Analysis Corresponding to the Limit State}

With regard to any engineering problems, it is extremely significant to determine the performance of structural limit state. Once time-varying uncertainties originating from inherent properties or external environmental conditions are considered, the limit state should have time-variant uncertainty as well. In fact, if we define the time-varying structural parameters as convex processes, the limit-state function can also be enclosed by a model of convex process. The detailed procedure of construction of the convex process model for structural limit state is mathematically discussed in the following statements.
A linear case of the limit-state function of the timevarying structure is expressed as

$$
g(t)=g(t, \mathbf{a}(t), \mathbf{X}(t))=a_{0}(t)+\sum_{i=1}^{n}\left(a_{i}(t) \cdot X_{i}(t)\right)
$$

where $\mathbf{X}(t)=\left(X_{1}(t), X_{2}(t), \ldots, X_{n}(t)\right)^{T}$ denotes a basic vector of convex processes, and $\mathbf{a}(t)=\left(a_{0}(t), a_{1}(t), a_{2}(t)\right.$, $\left.\ldots, a_{n}(t)\right)$ means a time-variant coefficient vector.

By virtue of the set-theory method, the mean value function and the radius function of limit-state function $g(t)$ are given by 


$$
\begin{aligned}
& g^{c}(t)=g^{c}(t, \mathbf{a}(t), \mathbf{X}(t))=a_{0}(t)+\sum_{i=1}^{n}\left(a_{i}(t) \cdot X_{i}^{c}(t)\right), \\
& g^{r}(t)=\sqrt{D_{g}(t)}=g^{r}(t, \mathbf{a}(t), \mathbf{X}(t))=\sqrt{\sum_{i=1}^{n}\left(a_{i}(t) \cdot X_{i}^{r}(t)\right)^{2}+\sum_{\substack{i=1 \\
j}}^{n} \sum_{\substack{j \neq i \\
j \neq i}}^{n} \rho_{X_{i} X_{j}}(t, t) \cdot a_{i}(t) \cdot a_{j}(t) \cdot X_{i}^{r}(t) \cdot X_{j}^{r}(t),}
\end{aligned}
$$

where $\mathbf{X}^{\mathrm{c}}(t)=\left(X_{1}^{c}(t), X_{2}^{c}(t), \ldots, X_{n}^{c}(t)\right)^{T}$ and $\mathbf{X}^{\mathbf{r}}(t)=\left(X_{1}^{r}(t)\right.$, $\left.X_{2}^{r}(t), \ldots, X_{n}^{r}(t)\right)^{T}$ are, respectively, the vectors of mean value function and the radius function for $\mathbf{X}(t), \rho_{X_{i} X_{j}}(t, t)$ stands for the cross-covariance function of variables $X_{i}(t)$ and $X_{j}(t)$, and $D_{g}(t)$ is the variance function.

In addition, taking into account the comprehensive effect of the autocorrelation between $X_{i}\left(t_{1}\right)$ and $X_{i}\left(t_{2}\right)$ as well as the cross-correlation between $X_{i}\left(t_{1}\right)$ and $X_{j}\left(t_{2}\right)$, the autocovariance function corresponding to $g\left(t_{1}\right)$ and $g\left(t_{2}\right)$ is found to be

$$
\begin{gathered}
\operatorname{Cov}_{g}\left(t_{1}, t_{2}\right)=\sum_{i=1}^{n} \rho_{X_{i}}\left(t_{1}, t_{2}\right) \cdot a_{i}\left(t_{1}\right) \cdot a_{i}\left(t_{2}\right) \cdot X_{i}^{r}\left(t_{1}\right) \\
\cdot X_{i}^{r}\left(t_{2}\right)+\sum_{\substack{i=1 \\
j}}^{n} \sum_{\substack{j \neq i \\
j \neq i}}^{n} \rho_{X_{i} X_{j}}\left(t_{1}, t_{2}\right) \cdot a_{i}\left(t_{1}\right) \cdot a_{j}\left(t_{2}\right) \\
\cdot X_{i}^{r}\left(t_{1}\right) \cdot X_{j}^{r}\left(t_{2}\right)
\end{gathered}
$$

where $\rho_{X}\left(t_{1}, t_{2}\right)$ is the autocorrelation coefficient function of $X_{i}\left(t_{1}\right)$ and $X_{i}\left(t_{2}\right)$ and $\rho_{X_{i} X_{j}}\left(t_{1}, t_{2}\right)$ is the cross-covariance function of $X_{i}\left(t_{1}\right)$ and $X_{j}\left(t_{2}\right)$.

In terms of the definitions in (5), we arrive at

$$
\rho_{g}\left(t_{1}, t_{2}\right)=\frac{\operatorname{Cov}_{g}\left(t_{1}, t_{2}\right)}{\sqrt{D_{g}\left(t_{1}\right)} \cdot \sqrt{D_{g}\left(t_{2}\right)}} .
$$

It is instructive to discuss the case of $g(R(t), S(t))=R(t)-$ $S(t)$, in which the strength $R(t)$ and the stress $S(t)$ are both enclosed by convex processes; we get

$$
\begin{aligned}
& \rho_{g}\left(t_{1}, t_{2}\right)=\left[R^{r}\left(t_{1}\right) \cdot R^{r}\left(t_{2}\right) \cdot \rho_{R}\left(t_{1}, t_{2}\right)+S^{r}\left(t_{1}\right) \cdot S^{r}\left(t_{2}\right)\right. \\
& \cdot \rho_{S}\left(t_{1}, t_{2}\right)-R^{r}\left(t_{1}\right) \cdot S^{r}\left(t_{2}\right) \cdot \rho_{R S}\left(t_{1}, t_{2}\right)-R^{r}\left(t_{2}\right) \cdot S^{r}\left(t_{1}\right) \\
& \left.\cdot \rho_{R S}\left(t_{2}, t_{1}\right)\right] \\
& \cdot \frac{1}{\sqrt{\left[\left(R^{r}\left(t_{1}\right)\right)^{2}+\left(S^{r}\left(t_{1}\right)\right)^{2}-2 R^{r}\left(t_{1}\right) \cdot S^{r}\left(t_{1}\right) \cdot \rho_{R S}\left(t_{1}, t_{1}\right)\right]}} \\
& \cdot \frac{1}{\sqrt{\left[\left(R^{r}\left(t_{2}\right)\right)^{2}+\left(S^{r}\left(t_{2}\right)\right)^{2}-2 R^{r}\left(t_{2}\right) \cdot S^{r}\left(t_{2}\right) \cdot \rho_{R S}\left(t_{2}, t_{2}\right)\right]}} .
\end{aligned}
$$

In accordance with the above equations, the time-varying uncertainty of limit-state $g(t)$ is explicitly embodied by a nonprobabilistic convex process model. That is to say, for any times $t_{1}$ and $t_{2}$, once the time-variant uncertainties existing in structural parameters are known, the ellipse utilized to restrict the feasible domain between $g\left(t_{1}\right)$ and $g\left(t_{2}\right)$ can be affirmed exclusively.

Apparently, it should be indicated that if the limitstate function is nonlinear, several linearization techniques, such as Taylor's series expansion and interval perturbation approach, may make effective contributions to help us construct its convex process model.

\section{Time-Dependent Reliability Measure Index and Its Solving Process}

4.1. The Classical First-Passage Method in Random Process Theory. For engineering problems, we are sometimes more interested in the calculation of time-varying probability/possibility of reliability because it provides us with the likelihood of a product performing its intended function over its service time. From the perspective of randomness, the time-dependent probability of failure/safety during a time interval $[0, T]$ is computed by

$$
P_{f}(T)=\operatorname{Pr}\{\exists t \in[0, T]: g(t, \mathbf{X}(t)) \leq 0\}
$$

or

$$
\begin{aligned}
R_{s}(T) & =1-P_{f}(T) \\
& =\operatorname{Pr}\{\forall t \in[0, T]: g(t, \mathbf{X}(t))>0\},
\end{aligned}
$$

where $\mathbf{X}(t)$ is a vector of random processes and $\operatorname{Pr}\{e\}$ stands for the probability of event $e$.

Generally, it is extremely difficult to obtain the exact solution of $P_{f}(T)$ or $R_{s}(T)$. At present, the most common approach to approximately solve time-dependent reliability problems in a rigorous way is the so-called first-passage approach (represented schematically in Figure 4).

The basic idea in first-passage approach is that crossing from the nonfailure into the failure domain at each instant time may be considered as being independent of one another. Denote by $N^{+}(0, T)$ the number of upcrossings of zero-value by the compound process $g(t, \mathbf{X}(t))$ from safe domain to the failure domain within $[0, T]$, and hence the probability of failure also reads

$$
P_{f}(T)=\operatorname{Pr}\left\{(g(0, \mathbf{X}(0))<0) \cup\left(N^{+}(0, T)>0\right)\right\} .
$$
that is,

Then, the following upper bound on $P_{f}(T)$ is available;

$$
P_{f}(T) \leq P_{f}(0)+\int_{0}^{T} \nu(t) d t
$$




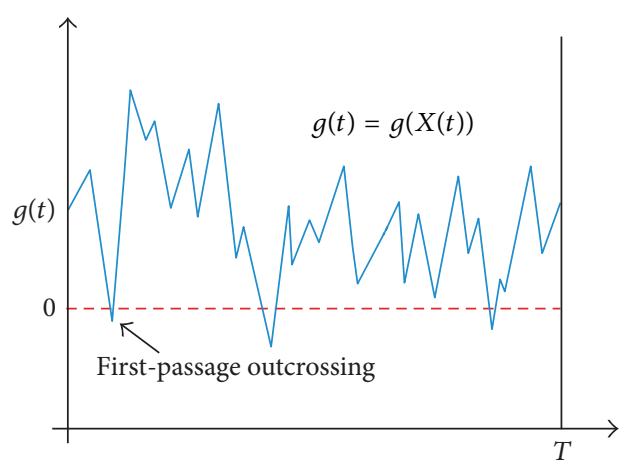

FIGURE 4: Schematic diagram for the first-passage approach.

where $P_{f}(0)$ is the probability of failure when time is fixed as zero and $\nu(t)$ is the instantaneous outcrossing rate at time $t$. The specific expression of $v(t)$ is evaluated as

$$
\begin{aligned}
& \nu(t) \\
& =\lim _{\Delta t \rightarrow 0} \frac{\operatorname{Pr}\{(g(t, \mathbf{X}(t))>0) \cap(g(t+\Delta t, \mathbf{X}(t+\Delta t))<0)\}}{\Delta t} .
\end{aligned}
$$

Introducing the finite-difference concept, rewrite (15) in the form

$$
\begin{aligned}
& v(t) \\
& \approx \frac{\operatorname{Pr}\{(g(t, \mathbf{X}(t))>0) \cap(g(t+\Delta t, \mathbf{X}(t+\Delta t))<0)\}}{\Delta t},
\end{aligned}
$$

where $\Delta t$ has to be selected properly (under the sufficiently small level).

4.2. Time-Dependent Reliability Measurement Based on Nonprobabilistic Convex Process Model. Although the firstpassage approach tends to provide a rigorous mathematical derivation of the time-dependent reliability, it is actually difficult to evaluate the outcrossing rate for the general random processes. Only in few cases, an analytical outcrossing rate is available. Furthermore, under the case of limited sample data of time-varying uncertainty, the analysis based on random process theory is inapplicable. Therefore, in this section, we apply the idea of the first-passage method into nonprobabilistic convex process model and further establish a new measure index for evaluating time-dependent reliability with insufficient information of uncertainty. The details are as follows:

(i) Acquire convex process corresponding to the limitstate $g(t)$ (by reference to the corresponding Sections 2 and 3$)$.

(ii) Discretize $g(t)$ into a time sequence; that is,

$$
\begin{array}{r}
g(0), g(\Delta t), g(2 \Delta t), \ldots, g(N \Delta t) \\
N=\frac{T}{\Delta t} \text { is sufficiently large. }
\end{array}
$$

(iii) Construct each ellipse model with respect to $g(i \Delta t)$ and $g((i+1) \Delta t)$, and further investigate the interference conditions between elliptic domain and event $E_{i}$ : $\{g(i \Delta t)>0 \cap g((i+1) \Delta t)<0\}$.

(iv) Redefine the outcrossing rate $\nu(i \Delta t)$ as

$$
\begin{aligned}
v(i \Delta t) & \approx \frac{\operatorname{Pos}\left\{E_{i}\right\}}{\Delta t} \\
& =\frac{\operatorname{Pos}\{(g(i \Delta t)>0) \cap(g((i+1) \Delta t)<0)\}}{\Delta t},
\end{aligned}
$$

where $\operatorname{Pos}\left\{E_{i}\right\}$ stands for the possibility of event $E_{i}$, which is regarded as the ratio of the interference area to the whole elliptic area $(i=1,2, \ldots, N)$; that is,

$$
\begin{aligned}
\operatorname{Pos}\left\{E_{i}\right\} & =\operatorname{Pos}\{(g(i \Delta t)>0) \cap(g((i+1) \Delta t)<0)\} \\
& =\frac{A_{\text {interference }}^{i}}{A_{\text {total }}^{i}} .
\end{aligned}
$$

(v) Determine the time-dependent reliability measure index; namely,

$$
\begin{aligned}
R_{s}(T) & =1-P_{f}(T) \\
& \geq 1-\left(\operatorname{Pos}(0)+\sum_{i=1}^{N}(\nu(i \Delta t) \cdot \Delta t)\right) \\
& =1-\left(\operatorname{Pos}(0)+\sum_{i=1}^{N}\left(\operatorname{Pos}\left\{E_{i}\right\}\right)\right) \\
& =1-\left(\operatorname{Pos}(0)+\sum_{i=1}^{N}\left(\frac{A_{\text {interference }}^{\mathrm{i}}}{A_{\text {total }}^{i}}\right)\right)
\end{aligned}
$$

4.3. Solution Strategy of Time-Dependent Reliability Measure Index. Obviously, the key point for determining the proposed time-dependent reliability measure index is just the calculation of $\operatorname{Pos}\left\{E_{i}\right\}$ (for practical problems of $\left(r_{2} / \sqrt{2}\right) g^{r}(t)<g^{c}(t)<g^{r}(t)$, as shown in Figure 5(a)). As mentioned above, the regularization methodology is firstly applied (as shown in Figure 5(b)), and the event $E_{i}$ is equivalent to

$$
\begin{aligned}
E_{i}: & \{g(i \Delta t)>0 \cap g((i+1) \Delta t)<0\} \Longrightarrow\left\{g^{c}(i \Delta t)\right. \\
& +g^{r}(i \Delta t) \cdot U_{1}>0 \cap g^{c}((i+1) \Delta t) \\
& \left.+g^{r}((i+1) \Delta t) \cdot U_{2}<0\right\} \Longrightarrow\left\{U_{1}>-\frac{g^{c}(i \Delta t)}{g^{r}(i \Delta t)}\right. \\
& \left.\cap U_{2}<-\frac{g^{c}((i+1) \Delta t)}{g^{r}((i+1) \Delta t)}\right\} .
\end{aligned}
$$

For the sake of convenience, one more coordinate transformation is then carried out, and a unit circular domain 


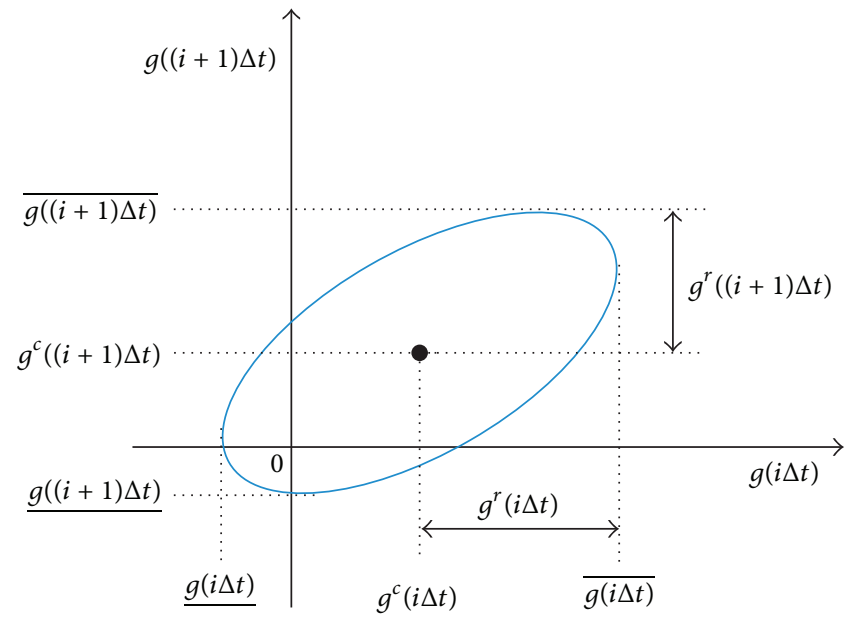

(a)

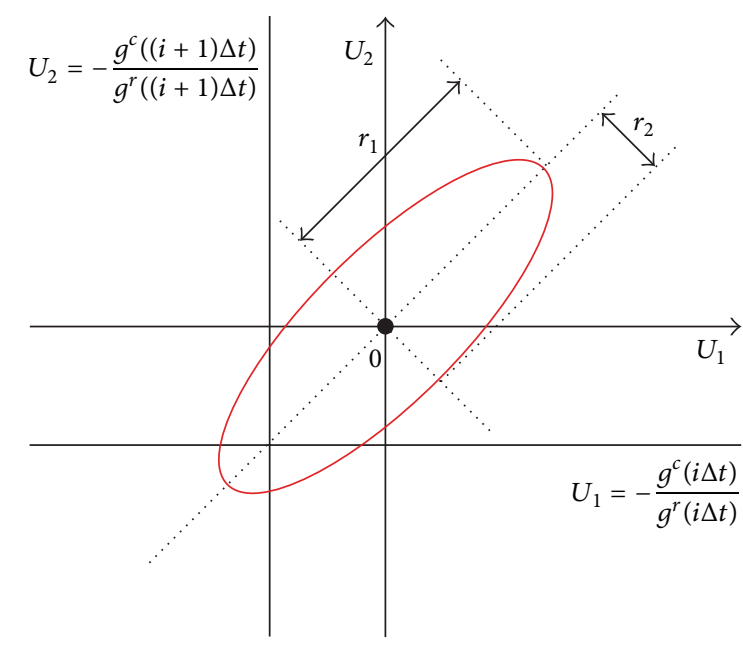

(b)

FIGURE 5: Uncertainty domains with regard to the limit-state function: (a) the original domain; (b) the regularized domain.

is eventually obtained (as shown in Figure 6). Equation (21) leads to

$$
\begin{aligned}
E_{i} & \Longrightarrow\left\{U_{1}>-\frac{g^{c}(i \Delta t)}{g^{r}(i \Delta t)} \cap U_{2}<-\frac{g^{c}((i+1) \Delta t)}{g^{r}((i+1) \Delta t)}\right\} \\
& \Longrightarrow\left\{r_{1} \zeta_{1} \cos \theta-r_{2} \zeta_{2} \sin \theta>-\frac{g^{c}(i \Delta t)}{g^{r}(i \Delta t)}\right. \\
& \left.\cap r_{1} \zeta_{1} \sin \theta+r_{2} \zeta_{2} \cos \theta<-\frac{g^{c}((i+1) \Delta t)}{g^{r}((i+1) \Delta t)}\right\} \\
& \Longrightarrow\left\{\zeta_{2}<\frac{r_{1}}{r_{2}} \zeta_{1}+\frac{\sqrt{2}}{r_{2}} \cdot \frac{g^{c}(i \Delta t)}{g^{r}(i \Delta t)} \cap \zeta_{2}<-\frac{r_{1}}{r_{2}} \zeta_{1}\right. \\
& \left.-\frac{\sqrt{2}}{r_{2}} \cdot \frac{g^{c}((i+1) \Delta t)}{g^{r}((i+1) \Delta t)}\right\} .
\end{aligned}
$$

From Figure 6, it becomes apparent that the shaded area $S_{B D E}$ indicates the interference area $A_{\text {interference }}^{i}$ and the total area $A_{\text {total }}^{i}$ always equals $\pi$. Hence, the analytical expression of $S_{B D E}$ is of paramount importance, and several following steps should be executed.

Step 1 (solution of the coordinates of points $A$ and $B$ ). Let us consider the simultaneous equations as

$$
\begin{aligned}
l_{1}: r_{2} \zeta_{2}-r_{1} \zeta_{1}-\sqrt{2} \frac{g^{c}(i \Delta t)}{g^{r}(i \Delta t)} & =0 \\
\zeta_{1} & =-\sqrt{1-\zeta_{2}^{2}} .
\end{aligned}
$$

By elimination method, a quadratic equation is further deduced; that is,

$$
\begin{aligned}
& \left(r_{1}^{2}+r_{2}^{2}\right) \cdot \zeta_{2}^{2}-2 \sqrt{2} r_{2} \cdot \frac{g^{c}(i \Delta t)}{g^{r}(i \Delta t)} \cdot \zeta_{2}+2\left(\frac{g^{c}(i \Delta t)}{g^{r}(i \Delta t)}\right)^{2} \\
& -r_{1}^{2}=0 .
\end{aligned}
$$

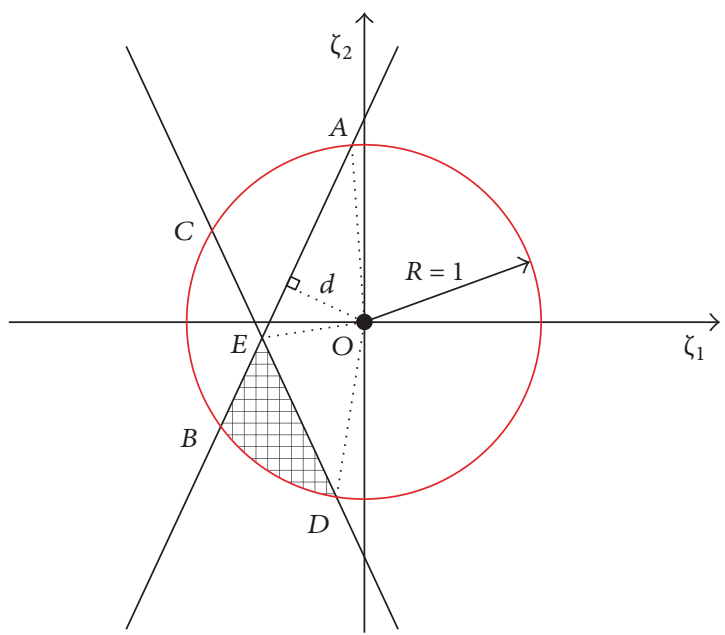

FIGURE 6: Schematic diagram for the solution of time-dependent reliability measure index.

Thus, the coordinates of points $A$ and $B$ on $\zeta_{2}$-axis are, respectively,

$$
\begin{aligned}
\zeta_{2 \_} A & =\frac{\sqrt{2}}{2}\left(r_{2} \cdot \frac{g^{c}(i \Delta t)}{g^{r}(i \Delta t)}\right. \\
& +\sqrt{\left.\left(r_{2}^{2}-2\right) \cdot\left(\frac{g^{c}(i \Delta t)}{g^{r}(i \Delta t)}\right)^{2}+r_{1}^{2}\right)}, \\
\zeta_{2 \_B} & =\frac{\sqrt{2}}{2}\left(r_{2} \cdot \frac{g^{c}(i \Delta t)}{g^{r}(i \Delta t)}\right. \\
& \left.-\sqrt{\left(r_{2}^{2}-2\right) \cdot\left(\frac{g^{c}(i \Delta t)}{g^{r}(i \Delta t)}\right)^{2}+r_{1}^{2}}\right) .
\end{aligned}
$$


Substituting (25) and (26) into (23), the coordinates of points $A$ and $B$ on $\zeta_{1}$-axis, namely, $\zeta_{1_{\perp} A}$ and $\zeta_{1 \_B}$, can be easily obtained.

Step 2 (solution of the coordinates of points $C$ and $D$ ). Taking into account the simultaneous equations

$$
\begin{aligned}
l_{2}: r_{2} \zeta_{2}+r_{1} \zeta_{1}+\sqrt{2} \frac{g^{c}((i+1) \Delta t)}{g^{r}((i+1) \Delta t)} & =0 \\
\zeta_{1} & =-\sqrt{1-\zeta_{2}^{2}},
\end{aligned}
$$

we arrive at

$$
\begin{aligned}
\left(r_{1}^{2}+\right. & \left.r_{2}^{2}\right) \cdot \zeta_{2}^{2}+2 \sqrt{2} r_{2} \cdot \frac{g^{c}((i+1) \Delta t)}{g^{r}((i+1) \Delta t)} \cdot \zeta_{2} \\
& +2\left(\frac{g^{c}((i+1) \Delta t)}{g^{r}((i+1) \Delta t)}\right)^{2}-r_{1}^{2}=0 .
\end{aligned}
$$

Therefore, the coordinates of points $C$ and $D$ on $\zeta_{2}$-axis are evaluated as

$$
\begin{aligned}
\zeta_{2 \_C} & =\frac{-\sqrt{2}}{2}\left(r_{2} \cdot \frac{g^{c}((i+1) \Delta t)}{g^{r}((i+1) \Delta t)}\right. \\
& \left.+\sqrt{\left(r_{2}^{2}-2\right) \cdot\left(\frac{g^{c}((i+1) \Delta t)}{g^{r}((i+1) \Delta t)}\right)^{2}+r_{1}^{2}}\right), \\
\zeta_{2 \_D} & =\frac{-\sqrt{2}}{2}\left(r_{2} \cdot \frac{g^{c}((i+1) \Delta t)}{g^{r}((i+1) \Delta t)}\right. \\
& \left.-\sqrt{\left(r_{2}^{2}-2\right) \cdot\left(\frac{g^{c}((i+1) \Delta t)}{g^{r}((i+1) \Delta t)}\right)^{2}+r_{1}^{2}}\right) .
\end{aligned}
$$

Substituting (29) and (30) into (27), the coordinates of points $C$ and $D$ on $\zeta_{1}$-axis, namely, $\zeta_{1_{-} C}$ and $\zeta_{1_{-} D}$, can be easily determined.

Step 3 (solution of the coordinates of point $E$ ). Since $E$ is actually the point of intersection between $l_{1}$ and $l_{2}$, the coordinate calculation is satisfied:

$$
\begin{array}{r}
l_{1}: r_{2} \zeta_{2}-r_{1} \zeta_{1}-\sqrt{2} \frac{g^{c}(i \Delta t)}{g^{r}(i \Delta t)}=0, \\
l_{2}: r_{2} \zeta_{2}+r_{1} \zeta_{1}+\sqrt{2} \frac{g^{c}((i+1) \Delta t)}{g^{r}((i+1) \Delta t)}=0 .
\end{array}
$$

Then we have

$$
\begin{aligned}
& \zeta_{1 \_E} \\
& =\frac{-\sqrt{2}\left(g^{c}(i \Delta t) / g^{r}(i \Delta t)+g^{c}((i+1) \Delta t) / g^{r}((i+1) \Delta t)\right)}{2 r_{1}}, \\
& \zeta_{2 \_E} \\
& =\frac{\sqrt{2}\left(g^{c}(i \Delta t) / g^{r}(i \Delta t)-g^{c}((i+1) \Delta t) / g^{r}((i+1) \Delta t)\right)}{2 r_{2}} .
\end{aligned}
$$

By virtue of the above three steps, the geometric information of the characteristic points (from $A$ to $E$ ) can be obtained mathematically, and the coordinates of these points are then used to deduce the analytical expression of $A_{\text {interference }}^{i}\left(S_{B D E}\right)$. In fact, the physical mean for definitions of the characteristic points lies in that the possibility of the cross failure during a small time interval can be eventually embodied by a form of geometric domain, which is determined by the points.

Step 4 (solution of the fan-shaped area $S_{0, \widehat{A C B D}}$ ). The central angel $\angle A O D$ is firstly computed by

$$
\angle A O D=\tan ^{-1}\left(\left|\frac{\zeta_{2_{-} A}}{\zeta_{1 \_A}}\right|\right)+\tan ^{-1}\left(\left|\frac{\zeta_{2 \_D}}{\zeta_{1 \_D}}\right|\right) .
$$

The area $S_{0 \_} \widehat{A C B D}$ equals

$$
\begin{aligned}
S_{0 \_} \overline{A C B D} & =\frac{1}{2} \angle A O D \cdot R^{2}=\frac{1}{2} \angle A O D \cdot 1^{2} \\
& =\frac{1}{2}\left(\tan ^{-1}\left(\left|\frac{\zeta_{2 \_A}}{\zeta_{1 \_A}}\right|\right)+\tan ^{-1}\left(\left|\frac{\zeta_{2 \_D}}{\zeta_{1 \_D}}\right|\right)\right),
\end{aligned}
$$

where $R$ represents the radius of the unit circular domain.

Step 5 (solution of the triangular areas $S_{\triangle A O E}$ and $S_{\triangle D O E}$ ). As previously noted, the coordinates of points $A, D$, and $E$ have been available. Thus, the triangular area $S_{\triangle A O E}$ is derived from

$$
\begin{aligned}
S_{\triangle A O E} & =\frac{1}{2}\left|\begin{array}{ccc}
1 & 1 & 1 \\
0 & \zeta_{1 \_A} & \zeta_{1 \_E} \\
0 & \zeta_{2 \_} & \zeta_{2 \_}
\end{array}\right| \\
& =\frac{1}{2}\left(\zeta_{1 \_A} \cdot \zeta_{2 \_E}-\zeta_{1 E} \cdot \zeta_{2 \_A}\right) .
\end{aligned}
$$

Similarly, $S_{\triangle D O E}$ is

$$
\begin{aligned}
S_{\triangle A O E} & =\frac{1}{2}\left|\begin{array}{ccc}
1 & 1 & 1 \\
0 & \zeta_{1 \_E} & \zeta_{1 \_D} \\
0 & \zeta_{2 E} & \zeta_{2 \_D}
\end{array}\right| \\
& =\frac{1}{2}\left(\zeta_{1 \_E} \cdot \zeta_{2 D}-\zeta_{1 D} \cdot \zeta_{2 \_E}\right) .
\end{aligned}
$$

Step 6 (solution of the bow-shaped area $S_{A C B}^{*}$ ). It can be found that the bow-shaped area $S_{A C B}^{\circ}$ is denoted by the difference with the fan-shaped area $S_{\overparen{0_{-} A C B}}$ and the triangular area $S_{\triangle A O B}$; namely,

$$
\begin{aligned}
S_{A C B}^{\bullet} & =S_{\overparen{0_{-} A C B}}-S_{\triangle A O B} \\
S_{\overparen{0_{-} A C B}} & =\frac{1}{2} \angle A O B \cdot R^{2}=\frac{1}{2} \angle A O B \cdot 1^{2} \\
& =\frac{1}{2} \cdot 2 \cos ^{-1} d \cdot 1^{2}=\cos ^{-1} d, \\
S_{\triangle A O B} & =\frac{1}{2} \overline{A B} \cdot d=\frac{1}{2} \cdot 2 \sqrt{1-d^{2}} \cdot d=d \sqrt{1-d^{2}},
\end{aligned}
$$

where $\overline{A B}$ is the chord length and $d$, as the distance from origin to line $l_{1}$, equals $g^{c}(i \Delta t) / g^{r}(i \Delta t)$. 


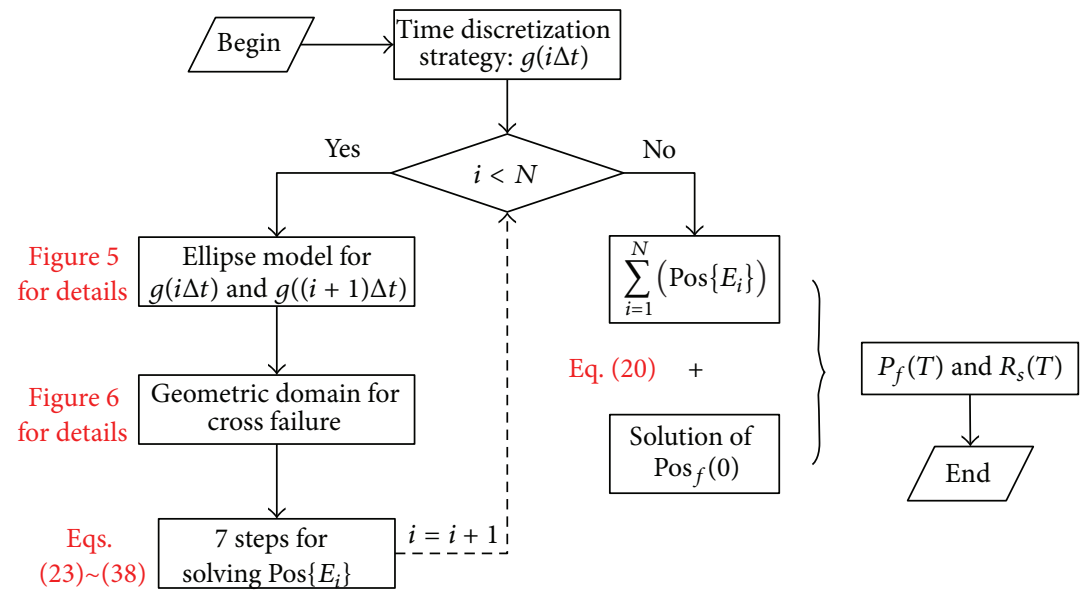

FIGURE 7: Flowchart of the time-dependent reliability analysis based on nonprobabilistic convex process model.

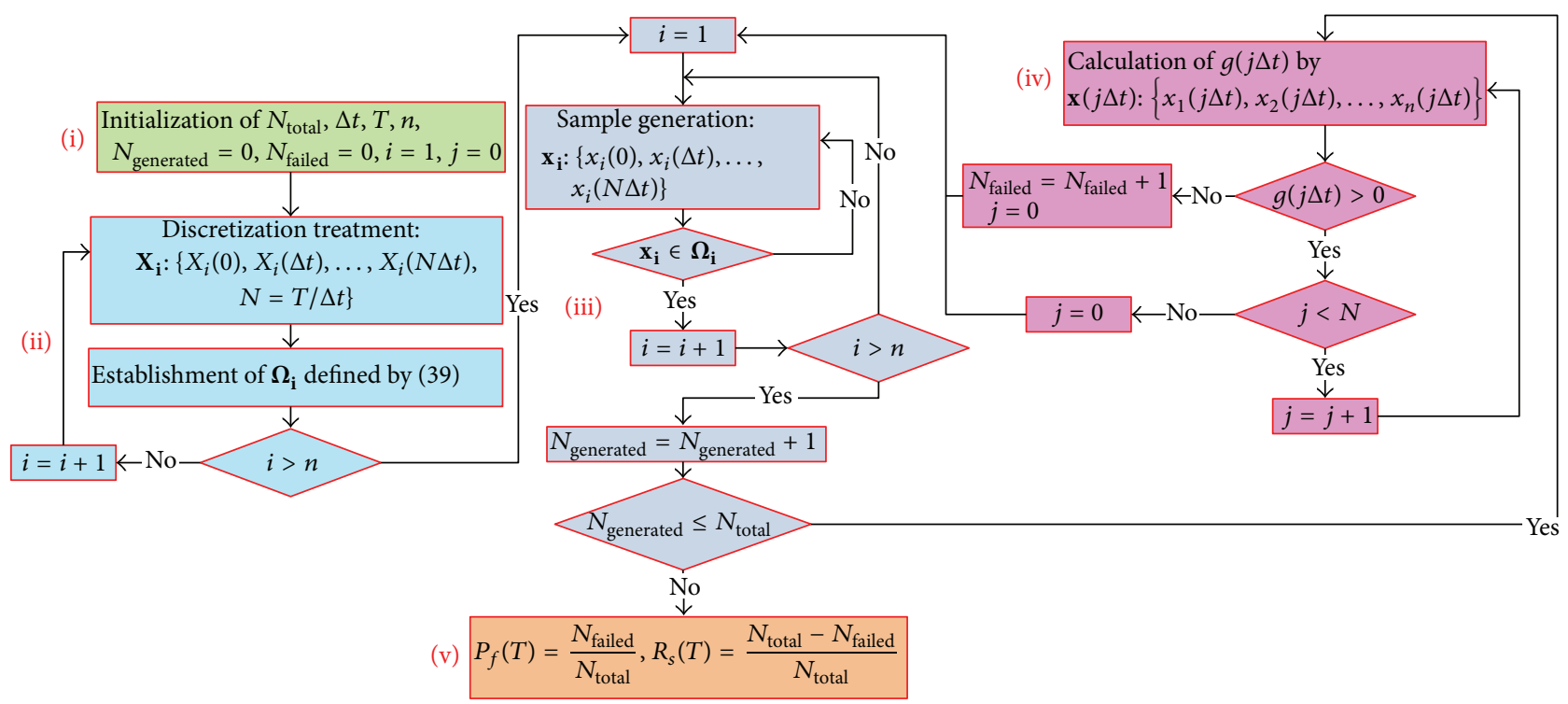

FIgURE 8: Flowchart of the time-dependent reliability analysis based on the Monte-Carlo simulation method.

Step 7 (solution of $\left.\operatorname{Pos}\left\{E_{i}\right\}\right)$. Consider

$$
\begin{aligned}
\operatorname{Pos}\left\{E_{i}\right\} & =\frac{A_{\text {interference }}^{i}}{A_{\text {total }}^{i}}=\frac{S_{B D E}}{\pi} \\
& =\frac{S_{0 \_\widehat{A C B D}}-S_{\triangle A O E}-S_{\triangle D O E}-S_{A C B}^{\bullet}}{\pi} .
\end{aligned}
$$

We should calculate $\operatorname{Pos}\left\{E_{i}\right\}$ successively $(i=1,2, \ldots, N)$ based on the above steps. By combination with the analysis stated in Section 4.2, an integral procedure for construction of the time-dependent reliability measure index can be eventually achieved. For ease of understanding, one flowchart is further added (see Figure 7 for details).

\section{Monte-Carlo Simulation Method}

For comparison's purpose, this paper also provides a MonteCarlo simulation method to compute structural timedependent reliability. The detailed analysis procedure is expounded below (as is seen in Figure 8):

(i) Initialize the numbers of generated samples and failed samples as $N_{\text {generated }}=0, N_{\text {failed }}=0$; define the total number of samples as $N_{\text {total }}$; the cyclic counting index $j$ is set to zero.

(ii) Assume a small time increment $\Delta t$, and discretize each convex process $X_{i}(t)$ as a time series $\mathbf{X}_{i}$ : $\left\{X_{i}(0), X_{i}(\Delta t), \ldots, X_{i}(N \Delta t), N=T / \Delta t\right\}$ in sequence $(i=1,2, \ldots, n)$; based on the characteristic properties of time-variant uncertainty, included by $X_{i}^{c}(t), X_{i}^{r}(t)$, and $\rho_{X_{i}}\left(t_{1}, t_{2}\right)$, construct a $(N+1)$-dimensional ellipsoidal domain $\Omega_{\mathbf{i}}$; that is, 


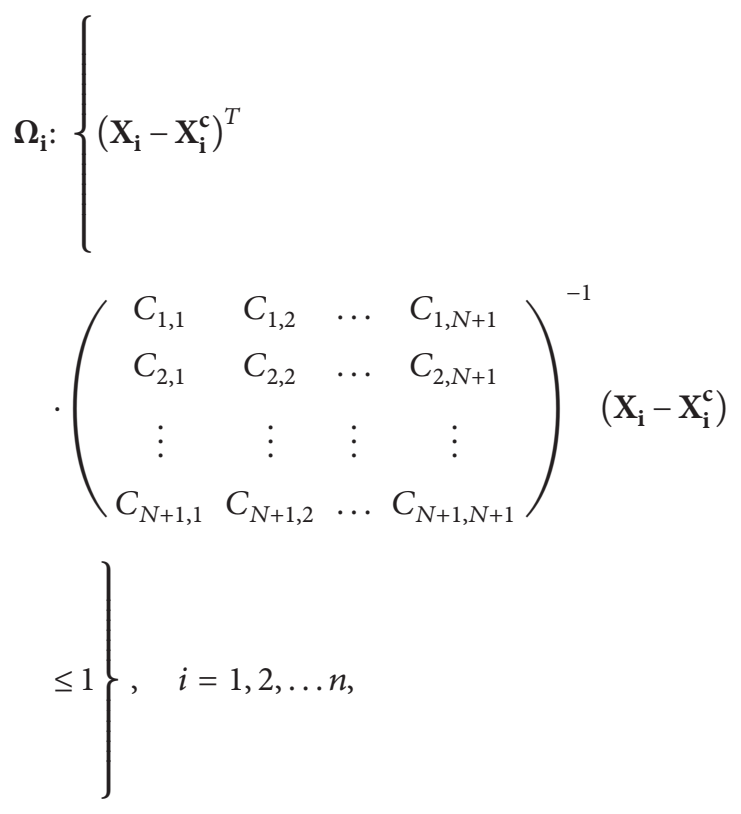

where $C_{l, s}=\operatorname{Cov}_{X_{i}}((l-1) \Delta t,(s-1) \Delta t)$.

(iii) According to the " $3 \sigma$ rule," generate one group of normal distributed samples $\mathbf{x}_{\mathbf{i}}:\left\{x_{i}(0), x_{i}(\Delta t), \ldots\right.$, $\left.x_{i}(N \Delta t)\right\}$ subjected to each time series $\mathbf{X}_{\mathbf{i}}(i=$ $1,2, \ldots, n)$; if $\mathbf{x}_{\mathbf{i}}$ could not meet the requirements of inequation from (39), restart (iii); otherwise, $N_{\text {generated }}=N_{\text {generated }}+1$, and then

(1) if $N_{\text {generated }} \leq N_{\text {total }}$, go to (iv);

(2) if not, go to (v).

(iv) Input the samples $\mathbf{x}(j \Delta t):\left\{x_{1}(j \Delta t), x_{2}(j \Delta t), \ldots\right.$, $\left.x_{n}(j \Delta t)\right\}$ into the limit-state function $g(j \Delta t)$ to estimate the structural safety at time $j \Delta t$; if $g(j \Delta t)>0$ and $j<N, j=j+1$, and restart (iv); else,

(1) if $j<N, N_{\text {failed }}=N_{\text {failed }}+1$, reset $j$ to zero and go back to (iii);

(2) if not, reset $j$ to zero and go back to (iii).

(v) The structural failure possibility is approximately computed as $P_{f}(T)=N_{\text {failed }} / N_{\text {total }}$; furthermore, the time-dependent reliability is obviously depicted as $R_{s}(T)=\left(N_{\text {total }}-N_{\text {failed }}\right) / N_{\text {total }}$.

By means of the proposed methodology of Monte-Carlo simulations, the numerical results of time-dependent reliability can be obtained in principle. However, some important issues remain unsolved in practical applications: (1) there is high complexity in sample generation for all convex processes, which is mainly embodied by the construction of multidimensional ellipsoidal convex models $\left(\boldsymbol{\Omega}_{1}, \boldsymbol{\Omega}_{2}, \ldots, \boldsymbol{\Omega}_{n}\right)$ under the case of small time increment $\Delta t$. (2) The above analysis is not able to demonstrate the cross-correlation between two convex processes. (3) The given assumption of " $3 \sigma$ rule" may result in an extremely large error when encountering the case of the combination of various uncertainty characteristics. (4) Enormous computational costs have to

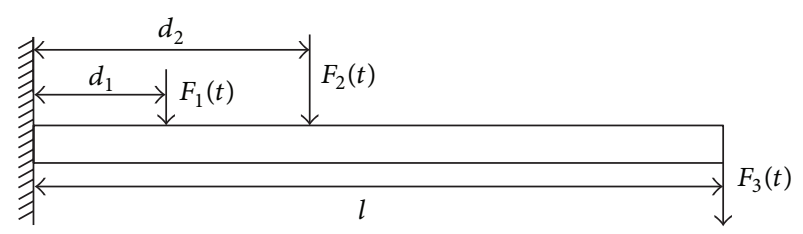

FIGURE 9: A cantilever beam structure.

be confronted when solving complex engineering problems. Therefore, compared with the Monte-Carlo simulations, the presented method based on nonprobabilistic convex process model may show superiority to some extent when dealing with the problems of time-dependent reliability evaluation.

\section{Numerical Examples}

In this section, three engineering examples are investigated. Among them, the first two examples of a cantilever beam structure and a ten-bar truss structure are further analyzed by Monte-Carlo simulations, and hence the numerical results can be regarded as a reference to effectively demonstrate the validity and feasibility of the presented methodology. That is to say, the accuracy of the proposed method is verified by the simulation techniques, and the deviation of the reliability results may quantify a specific precision level.

Additionally, the safety estimation of a complicated propeller structure in the last example can better illustrate the advantage and capability of the developed time-dependent reliability method when tackling reliability issues of large complex structures.

6.1. A Cantilever Beam Structure. A cantilever beam structure modified from the numerical example in [28] is considered, as shown in Figure 9. Three time-varying external forces $F_{1}(t)$, $F_{2}(t)$, and $F_{3}(t)$ are applied to the beam, and the maximum moment on the constraint surface at the origin should be less than an allowable value $M_{\text {criteria }}(t)$. Thus, the following limitstate function can be created by

$$
\begin{aligned}
g(t)= & g\left(F_{1}(t), F_{2}(t), F_{3}(t), M_{\text {criteria }}(t)\right) \\
= & M_{\text {criteria }}(t)-d_{1} \cdot F_{1}(t)-d_{2} \cdot F_{2}(t)-l \\
& \cdot F_{3}(t), \quad t \in[0, T],
\end{aligned}
$$

where $d_{1}=1 \mathrm{~m}, d_{2}=2 \mathrm{~m}$, and $l=5 \mathrm{~m}$ and $T$ in this problem is defined as 10 years.

Here, $F_{1}(t), F_{2}(t), F_{3}(t)$, and $M_{\text {criteria }}(t)$ are treated as convex processes containing autocorrelation but without consideration of cross-correlation. All the uncertainty properties are summarized in Table 1 where $\alpha=1,1.2,1.4,1.6,1.8,2$ and $k=1,2,3,4,5$. Various combinations of $\alpha$ and $k$ embody different spans and autocorrelation effects of convex processes.

The time-dependent reliability results $R_{s}(T)$ obtained by the nonprobabilistic convex process model are given in Table 2 and Figure 10 ( $\Delta t=0.05$ years). It can be found that values of $R_{s}(T)$ decrease remarkably when either $\alpha$ or $k$ 
TABLE 1: Time-varying uncertainty characteristics of the cantilever beam structure.

\begin{tabular}{lccc}
\hline & Mean value functions and radius functions of convex processes: $F_{1}(t), F_{2}(t), F_{3}(t), M_{\text {criteria }}(t)$ \\
\hline$F_{1}^{c}(t)=\frac{1}{2} \sin \left(4 \pi t+\frac{\pi}{4}\right)+\frac{5}{2}$ & $F_{2}^{c}(t)=-\cos \left(8 \pi t-\frac{\pi}{3}\right)+5$ & $F_{3}^{c}(t)=-\frac{3}{4} \cos \left(6 \pi t+\frac{2 \pi}{5}\right)+\frac{3}{2}$ & $M_{\text {criteria }}^{c}(t)=28-\frac{1}{5} t$ \\
$F_{1}^{r}(t)=F_{1}^{c}(t) \times 6 \alpha \%$ & $F_{2}^{r}(t)=F_{2}^{c}(t) \times 5 \alpha \%$ & $F_{3}^{r}(t)=F_{3}^{c}(t) \times 3 \alpha \%$ & $M_{\text {criteria }}^{r}(t)=\frac{\alpha t^{2}}{100}$ \\
(unit: $)$ & (unit: $)$ & (unit: $)$ & (unit: $\mathrm{N} \cdot \mathrm{m})$
\end{tabular}

\begin{tabular}{ccc}
\hline Correlation coefficient functions of convex processes: $F_{1}(t), F_{2}(t), F_{3}(t), M_{\text {criteria }}(t)$ \\
\hline$\rho_{F_{1}}\left(t_{1}, t_{2}\right)=e^{-\left(4 k \cdot\left|t_{1}-t_{2}\right|\right)}$ & $\rho_{F_{2}}\left(t_{1}, t_{2}\right)=e^{-\left(5 k \cdot\left|t_{1}-t_{2}\right|\right)} \cdot \cos \left(k \cdot\left|t_{1}-t_{2}\right|\right)$ & $\begin{array}{l}\text { No consideration of } \\
\text { cross-correlation }\end{array}$ \\
$\rho_{F_{3}}\left(t_{1}, t_{2}\right)=e^{-\left((5 / 2) k \cdot\left|t_{1}-t_{2}\right|\right)^{2}}$ & $\rho_{M_{\text {criteria }}}\left(t_{1}, t_{2}\right)=\frac{1}{2}\left(1+\cos \left(6 k \cdot\left|t_{1}-t_{2}\right|\right)\right)$ & con \\
\hline
\end{tabular}

TABLE 2: Reliability results based on the nonprobabilistic convex process model.

\begin{tabular}{lcccccc}
\hline$k / \alpha$ & $\alpha=1.0$ & $\alpha=1.2$ & $\alpha=1.4$ & $\alpha=1.6$ & $\alpha=1.8$ & $\alpha=2.0$ \\
\hline$k=1$ & 1 & 0.986 & 0.9426 & 0.8931 & 0.8259 & 0.7595 \\
$k=2$ & 1 & 0.9857 & 0.9424 & 0.8881 & 0.8155 & 0.7425 \\
$k=3$ & 1 & 0.9854 & 0.9423 & 0.8821 & 0.8027 & 0.7249 \\
$k=4$ & 1 & 0.9853 & 0.9419 & 0.8741 & 0.7915 & 0.7085 \\
$k=5$ & 1 & 0.9852 & 0.9406 & 0.8696 & 0.7844 & 0.6972 \\
\hline
\end{tabular}

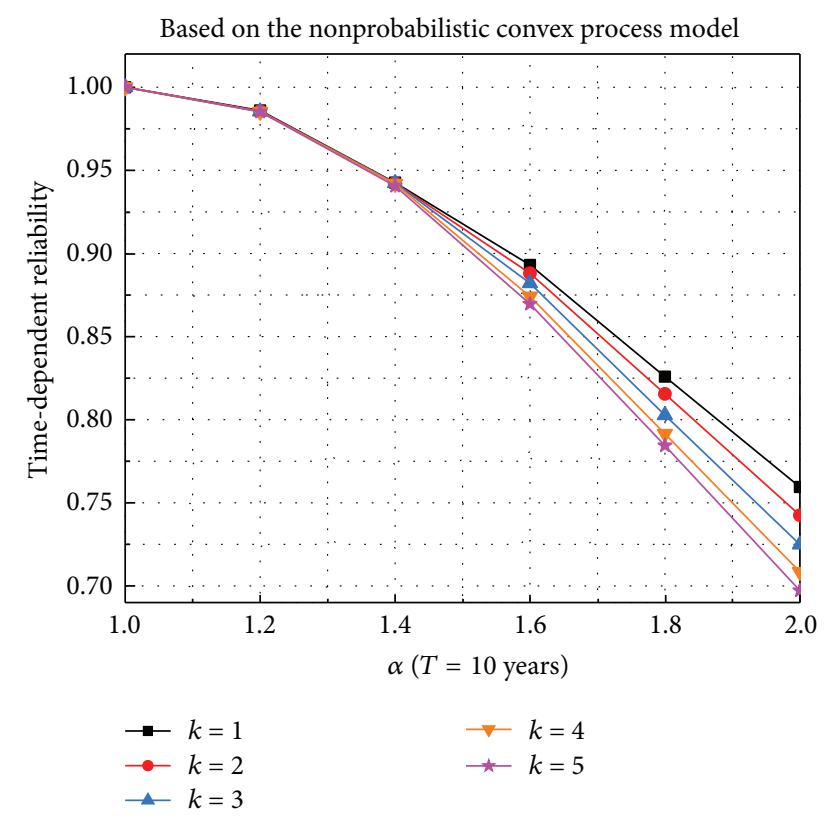

Figure 10: Time-dependent reliability results versus $\alpha$ and $k$.

increases, as expected. This indicates that higher dispersion or weaker correlation leads to lower structural safety. For example, the cantilever beam is absolutely safe when $\alpha$ and $k$ are both equal to 1 , while $R_{s}(T)$ only reaches 0.6972 under the case of $\alpha=2$ and $k=5$.

To better analyze the accuracy of the time-dependent reliability results, the Monte-Carlo simulation method is also used to deal with a severe case of $\alpha=2$ (100000 samples). The comparative results are illustrated in Figure 11. As shown in Figure 11, $R_{s}(T)$ calculated by our method is coincident with the results derived from the numerical simulations. However,

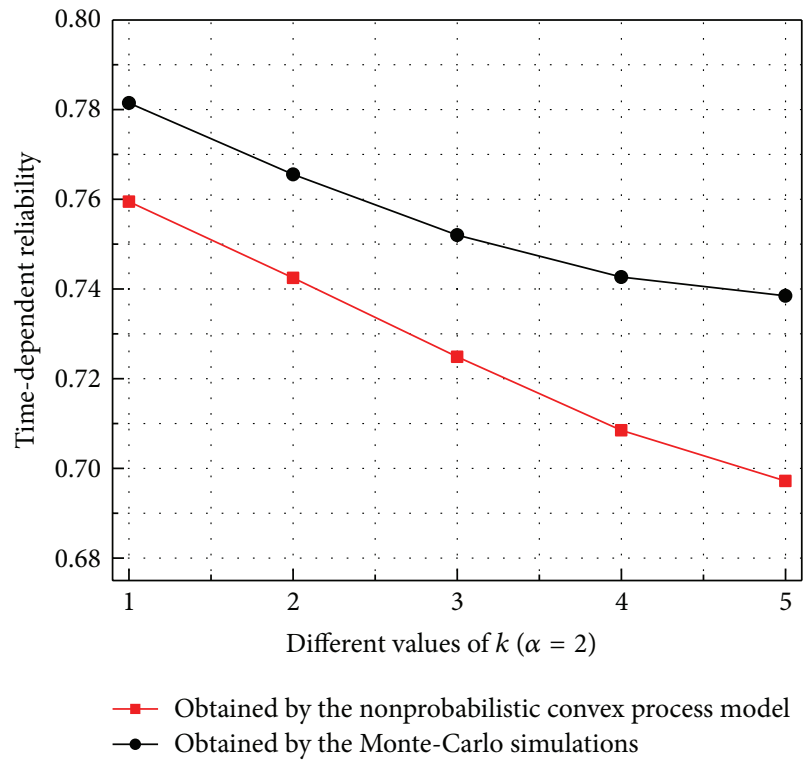

FIGURE 11: Comparisons of the reliability results obtained by the convex process model and the Monte-Carlo based model.

there are three points that should be noted: (1) The results based on convex process model are more conservative due to the fact that less information on time-varying uncertainty is needed. (2) Associated with the increasing values of $k$, the deviation of the reliability results $R_{s}(T)$ is widening (from $2.90 \%$ to $5.92 \%$ ). (3) The accuracy of the reliability from Monte-Carlo simulations mainly relies on the number of samples which implies that the situation of intensive computation and consuming time may have to be confronted.

6.2. A Ten-Bar Truss Structure. A well-known ten-bar truss structure modified from the numerical example in [15] is investigated, as depicted in Figure 12. Young's modulus $E=$ $68948 \mathrm{MPa}$, and the density $\rho=7.85 \times 10^{-9} \mathrm{t} / \mathrm{mm}^{3}$. The length $l$ of the horizontal and vertical bars is $9144 \mathrm{~mm}$, and the area of each bar equals $4000 \mathrm{~mm}^{2}$. Subjected to two timevarying vertical forces, $F_{1}(t)$ and $F_{2}(t)$, and one time-varying horizontal force, $F_{3}(t)$, the structure is defined as failure if $S_{T, \text { max }}(t) \geq R_{T, \text { criteria }}(t)$ or $\left|S_{C, \text { max }}(t)\right| \geq\left|R_{C \text {, criteria }}(t)\right|$ occurs, where $S_{T, \max }(t)$ and $S_{C, \max }(t)$ stand for the maximal values of tensile stress and compressive stress among all the members; 
TABLE 3: Time-varying uncertainty characteristics of the ten-bar truss structure.

\begin{tabular}{|c|c|c|c|}
\hline \multicolumn{4}{|c|}{ Mean value functions and radius functions of convex processes: $F_{1}(t), F_{2}(t), F_{3}(t), R_{T, \text { criteria }}(t), R_{C, \text { criteria }}(t)$} \\
\hline$F_{1}^{c}(t)=F_{2}^{c}(t)=2 \sin \left(3 \pi t+\frac{\pi}{3}\right)+44.48$ & $F_{3}^{c}(t)=7 \cos \left(4 \pi t-\frac{\pi}{5}\right)+177.92$ & $R_{T, \text { criteria }}^{c}(t)=375-\frac{15 t}{8}$ & $R_{T, \text { criteria }}^{r}(t)=\frac{\alpha t^{2}}{36}$ \\
\hline $\begin{array}{l}F_{1}^{r}(t)=F_{2}^{r}(t)=F_{1}^{c}(t) \times 3 \alpha \% \\
\text { (unit: } \mathrm{KN})\end{array}$ & $\begin{array}{c}F_{3}^{r}(t)=F_{3}^{c}(t) \times 2 \alpha \% \\
\quad(\text { unit: KN) }\end{array}$ & \multicolumn{2}{|c|}{$\begin{array}{c}R_{C, \text { criteria }}^{c}(t)=-100+\frac{3 t}{4} \quad R_{C, \text { criteria }}^{r}(t)=\frac{\alpha t^{2}}{32} \\
\text { (unit: } \mathrm{MPa})\end{array}$} \\
\hline \multicolumn{4}{|c|}{ Correlation coefficient functions of convex processes: $F_{1}(t), F_{2}(t), F_{3}(t), R_{T, \text { criteria }}(t), R_{C, \text { criteria }}(t)$} \\
\hline 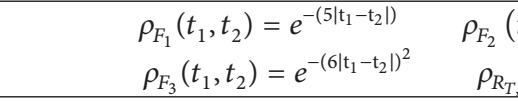 & $\begin{array}{l}=e^{-\left(5\left|t_{1}-t_{2}\right|\right)} \cdot \cos \left(4\left|t_{1}-t_{2}\right|\right) \\
\left.t_{1}, t_{2}\right)=\rho_{R_{C, \text { rriteria }}}\left(t_{1}, t_{2}\right)=0\end{array}$ & \multicolumn{2}{|c|}{$\begin{array}{l}\text { Taking into account the cross-correlation between } \\
F_{1}(t) \text { and } F_{2}(t)\end{array}$} \\
\hline
\end{tabular}

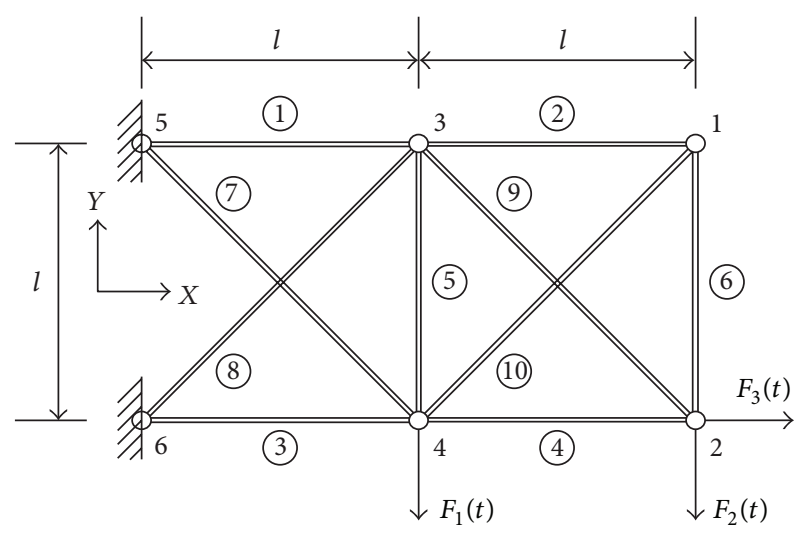

FIGURE 12: A ten-bar truss structure.

$R_{T, \text { criteria }}(t)$ and $R_{C \text {,criteria }}(t)$ are, respectively, the allowable values at time $t$ under the state of tension and compression. Hence, the following two limit-state functions are expressed as

$$
\begin{aligned}
& g_{1}(t)=g\left(S_{T, \max }(t), R_{T, \text { criteria }}(t)\right) \\
& =R_{T, \text { criteria }}(t)-S_{T, \text { max }}\left(F_{1}(t), F_{2}(t), F_{3}(t)\right) \text {, } \\
& t \in[0, T], \\
& g_{2}(t)=g\left(S_{C, \max }(t), R_{C, \text { criteria }}(t)\right) \\
& =\left|R_{C, \text { criteria }}(t)\right| \\
& -\left|S_{C, \max }\left(F_{1}(t), F_{2}(t), F_{3}(t)\right)\right| \text {, } \\
& t \in[0, T],
\end{aligned}
$$

where $T=10$ years.

In this problem, $F_{1}(t), F_{2}(t), F_{3}(t), R_{T, \text { criteria }}(t)$, and $R_{C \text {,criteria }}(t)$ are defined as convex processes, where the autocorrelation and the cross-correlation between $F_{1}(t)$ and $F_{2}(t)$ are simultaneously considered. The uncertainty properties of the above convex processes are given in Table 3 where $\alpha=$ $1.5,1.6,1.7,1.8,1.9,2$ and the cross-correlation coefficient $\rho_{F_{1} F_{2}}=-0.2,0.1,0,0.1,0.2$.

It is not difficult to understand that member 4 and member 8 are most dangerous, respectively, under the tensile situation and compressive situation. According to the definitions in (41) and (42), the time-dependent reliability results $R_{s}(T)$ obtained by the nonprobabilistic convex process
TABLE 4: Reliability results based on the nonprobabilistic convex process model (member 4).

\begin{tabular}{lccccccc}
\hline$\rho_{F_{1} F_{2}} / \alpha$ & $\alpha=1.5$ & $\alpha=1.6$ & $\alpha=1.7$ & $\alpha=1.8$ & $\alpha=1.9$ & $\alpha=2.0$ \\
\hline$\rho_{F_{1} F_{2}}=-0.2$ & 0.9096 & 0.873 & 0.8329 & 0.7904 & 0.7369 & 0.6714 \\
$\rho_{F_{1} F_{2}}=-0.1$ & 0.9085 & 0.8704 & 0.8319 & 0.7873 & 0.7327 & 0.6647 \\
$\rho_{F_{1} F_{2}}=0$ & 0.906 & 0.8684 & 0.8302 & 0.7842 & 0.7284 & 0.6635 \\
$\rho_{F_{1} F_{2}}=0.1$ & 0.9053 & 0.8675 & 0.8296 & 0.7821 & 0.7272 & 0.659 \\
$\rho_{F_{1} F_{2}}=0.2$ & 0.9047 & 0.8669 & 0.8276 & 0.7805 & 0.7238 & 0.6579 \\
\hline
\end{tabular}

TABLE 5: Reliability results based on the nonprobabilistic convex process model (member 8 ).

\begin{tabular}{lccccccc}
\hline$\rho_{F_{1} F_{2}} / \alpha$ & $\alpha=1.5$ & $\alpha=1.6$ & $\alpha=1.7$ & $\alpha=1.8$ & $\alpha=1.9$ & $\alpha=2.0$ \\
\hline$\rho_{F_{1} F_{2}}=-0.2$ & 1 & 0.9947 & 0.9581 & 0.8928 & 0.7899 & 0.6521 \\
$\rho_{F_{1} F_{2}}=-0.1$ & 0.99941 & 0.9817 & 0.929 & 0.8385 & 0.7075 & 0.5658 \\
$\rho_{F_{1} F_{2}}=0$ & 0.9954 & 0.9603 & 0.8888 & 0.7762 & 0.633 & 0.482 \\
$\rho_{F_{1} F_{2}}=0.1$ & 0.9859 & 0.935 & 0.8405 & 0.7057 & 0.5589 & 0.4028 \\
$\rho_{F_{1} F_{2}}=0.2$ & 0.9706 & 0.9003 & 0.7858 & 0.6381 & 0.4884 & 0.3216 \\
\hline
\end{tabular}

model are given in Tables 4 and 5 and Figures 13 and 14 ( $\Delta t=0.05$ years). It is remarkable that (1) the results of $R_{s}(T)$ continue to decline as the increase of $a$. (2) If $\rho_{F_{1} F_{2}}$ is negative, stronger cross-correlation may lead to a higher level of structural safety; if $\rho_{F_{1} F_{2}}$ is positive, however, the truss will be more dangerous with an increasing $\rho_{F_{1} F_{2}}$. (3) $\rho_{F_{1} F_{2}}$ has a minor effect on reliability results for member 4 , while it plays an important role in safety estimation for member 8 .

For the purpose of verification and comparison, the Monte-Carlo simulation method is utilized again to analyze the case of $\rho_{F_{1} F_{2}}=0$ (in disregard of cross-correlation). The time-dependent reliability results are illustrated in Figures 15 and 16. Similarly to conditions on the first example, the timedependent reliability results calculated by convex process model are consistent with the results computed by the MonteCarlo simulation method in qualitative analysis but lower to some extent from quantitative perspective. Specifically, when $a=2$, the maximal deviations of reliability results are, respectively, $13.06 \%$ for member 4 and $26.17 \%$ for member 8 .

6.3. A Marine Propeller Structure. The high-speed rotating propeller structure, as a vital component of power plant, must reach high standard of safety during its whole lifetime 


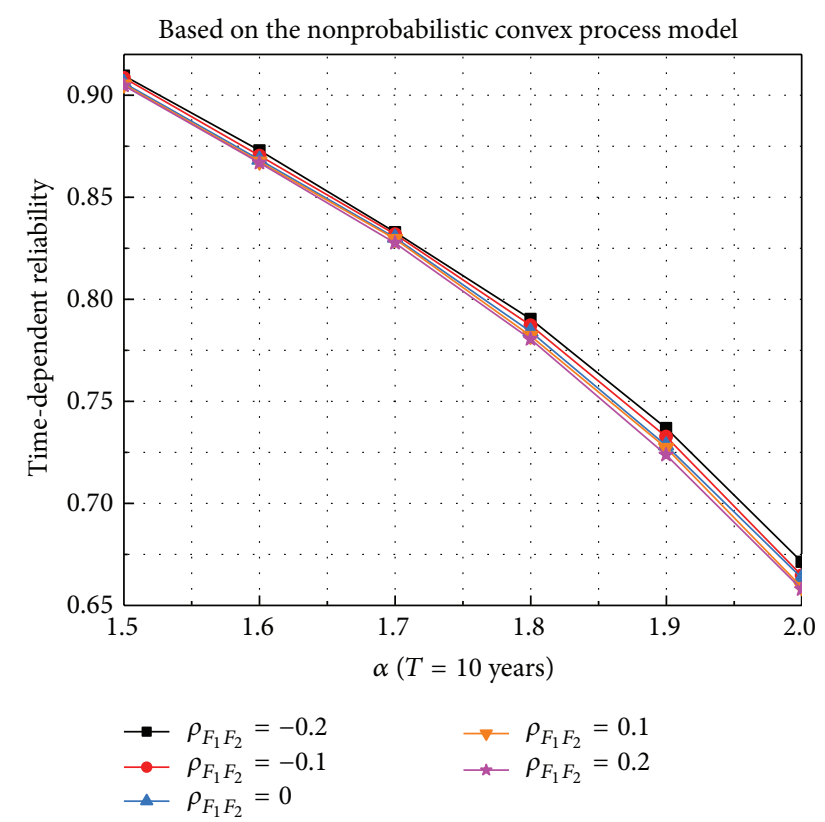

FIGURE 13: Time-dependent reliability results versus $\alpha$ and $\rho_{F_{1} F_{2}}$ (member 4).

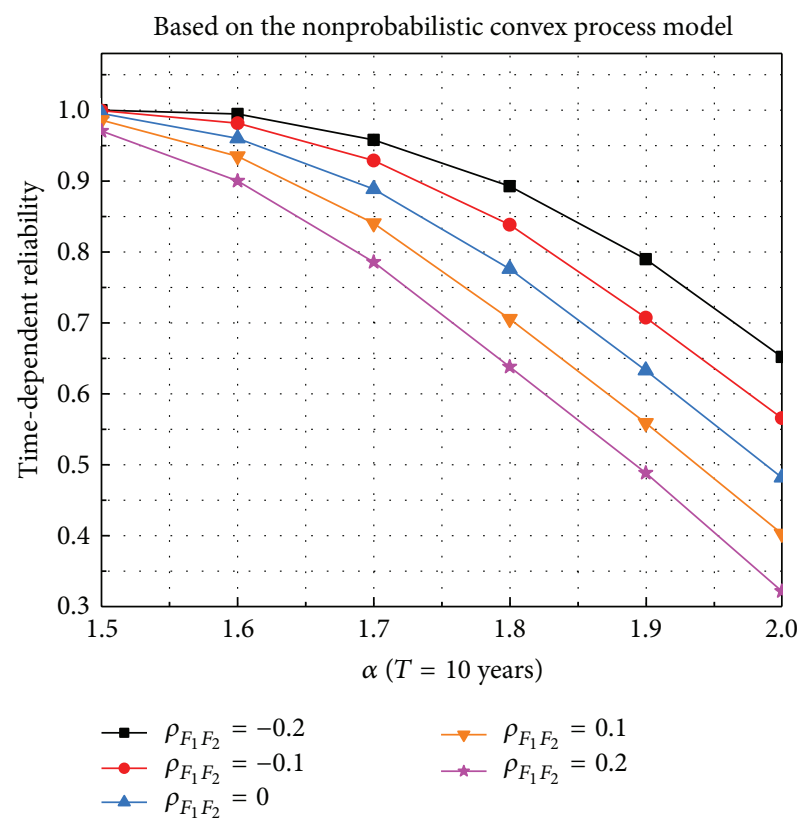

FIgURE 14: Time-dependent reliability results versus $\alpha$ and $\rho_{F_{1} F_{2}}$ (member 8).

to guarantee normal operations of marine system. In actual working conditions, affected by changeable environment of water flow, the forces experienced on propeller are always complicated and are uncertain at different times. Therefore, the time-dependent reliability analysis of this structure is of great significance. Figure 17 shows a specific type of propeller, which is composed of four blades and one support block, and its material parameters are listed in Table 6. Three types of time-varying forces are applied to the surface of each blade,

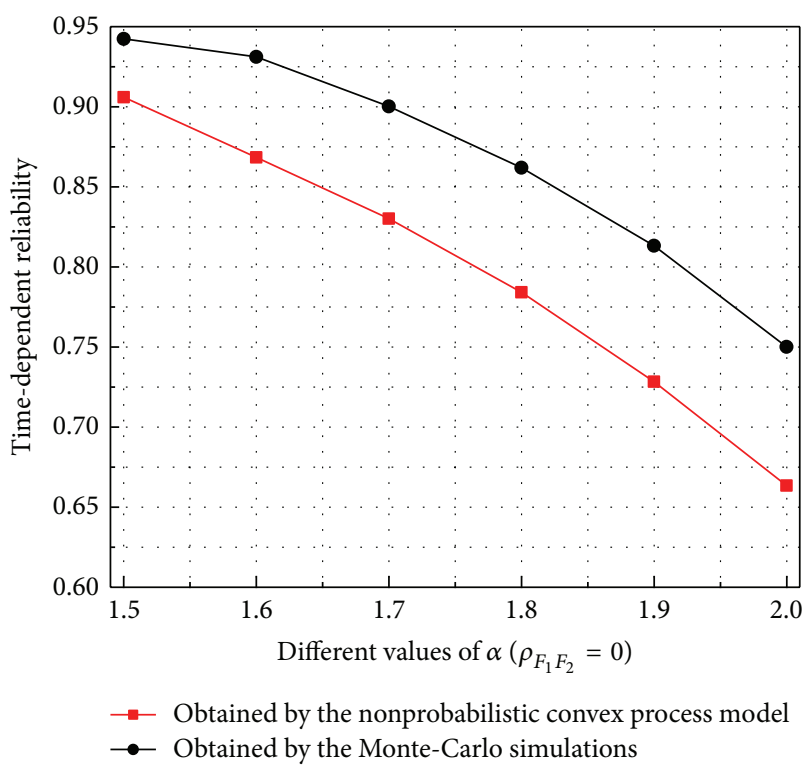

Figure 15: Comparisons of the reliability results obtained by the convex process model and the Monte-Carlo based model (member $4)$.

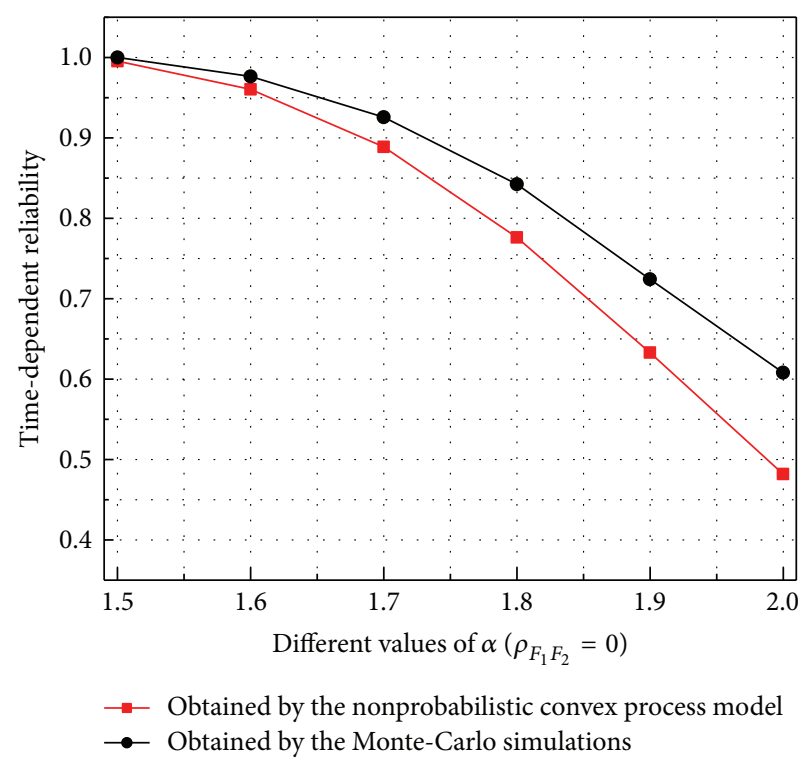

FIGURE 16: Comparisons of the reliability results obtained by the convex process model and the Monte-Carlo based model (member 8).

namely, the propulsive force $F_{1}(t)$, the centrifugal force $F_{2}(t)$, and the shear force $F_{3}(t)$. Hence, the limit-state function is expressed as

$$
\begin{aligned}
g(t) & =g\left(\sigma_{\max }(t), \sigma_{\text {criteria }}(t)\right) \\
& =\sigma_{\text {criteria }}(t)-\sigma_{\max }\left(F_{1}(t), F_{2}(t), F_{3}(t)\right), \\
& t \in[0, T],
\end{aligned}
$$



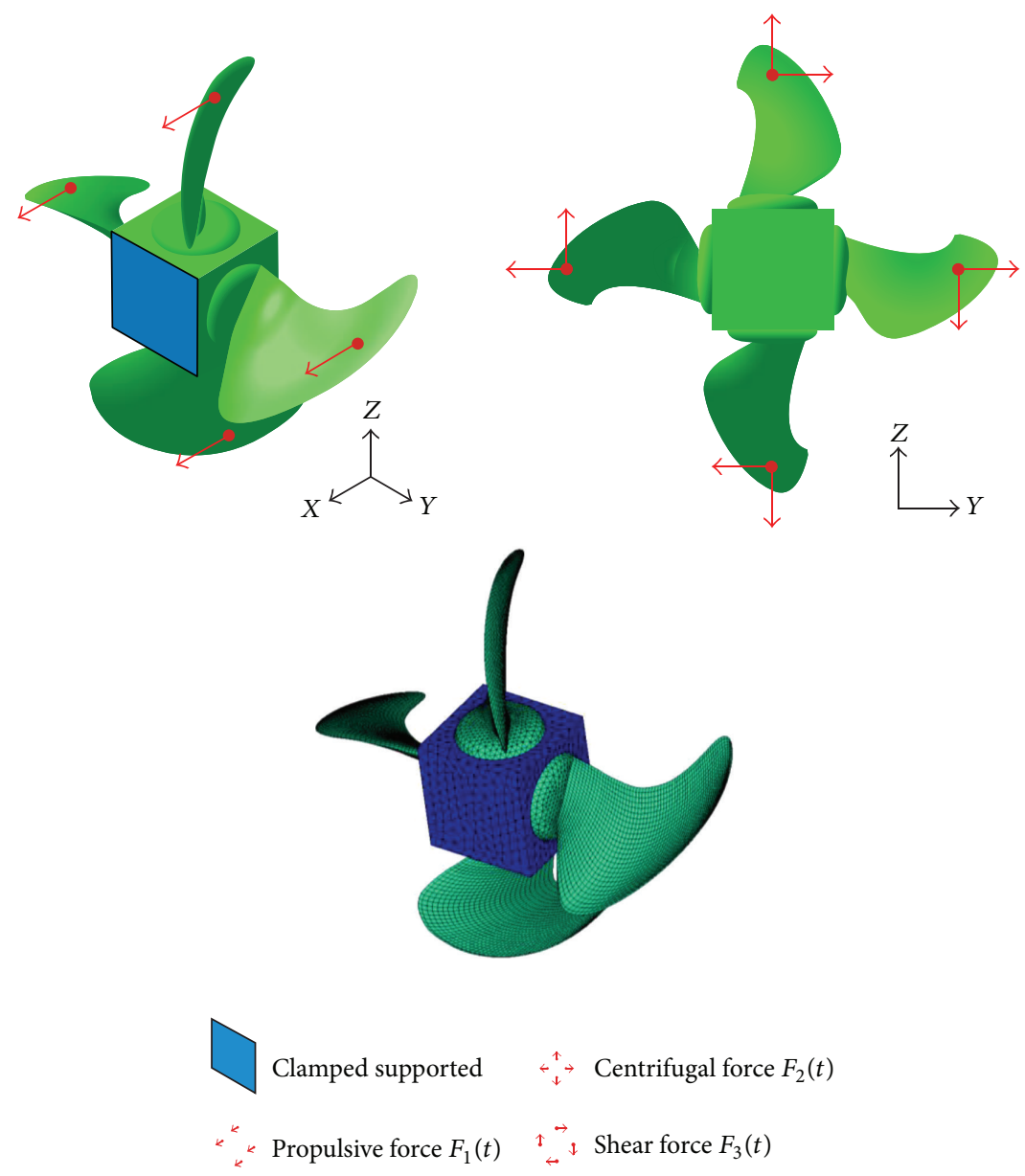

FIgURE 17: A marine propeller structure.

TABLE 6: Material parameters of the marine propeller structure.

\begin{tabular}{lcc}
\hline Young's modulus $E$ & Poisson's ratio $v$ & Density $\rho$ \\
\hline $2 \times 10^{5} \mathrm{MPa}$ & 0.3 & $7.8 \times 10^{3} \mathrm{Kg} / \mathrm{m}^{3}$ \\
\hline
\end{tabular}

where $\sigma_{\max }(t)$ denotes the maximum von Mises stress of the propeller structure, $\sigma_{\text {criteria }}(t)$ is allowable strength, and $T=10$ years. Considering that the propeller belongs to a complicated three-dimensional structure, the dynamic finite element model, containing 44888 elements and 31085 nodes, is set up to estimate the structural safety.

Assume that dynamic forces $F_{1}(t), F_{2}(t)$, and $F_{3}(t)$ as well as allowable strength $\sigma_{\text {criteria }}(t)$ are all convex processes, where autocorrelation and cross-correlation are both considered. The detailed information of time-varying uncertainty is summarized in Table 7 where $\alpha$ can be valued by $0.1,0.2, \ldots, 1$.

The present method is applied to the above engineering problem, and all the analysis results are given in Table 8 and Figure 18 ( $\Delta t=0.05$ years). It can be seen that (1) there is no need to worry about failure when $\alpha=0.1$ or $0.2\left(R_{s}(T)=1\right)$. (2) As $\alpha$ increases, the time-dependent reliability may show a decreasing trend (the minimum $R_{s}(T)=0.7538$ under the

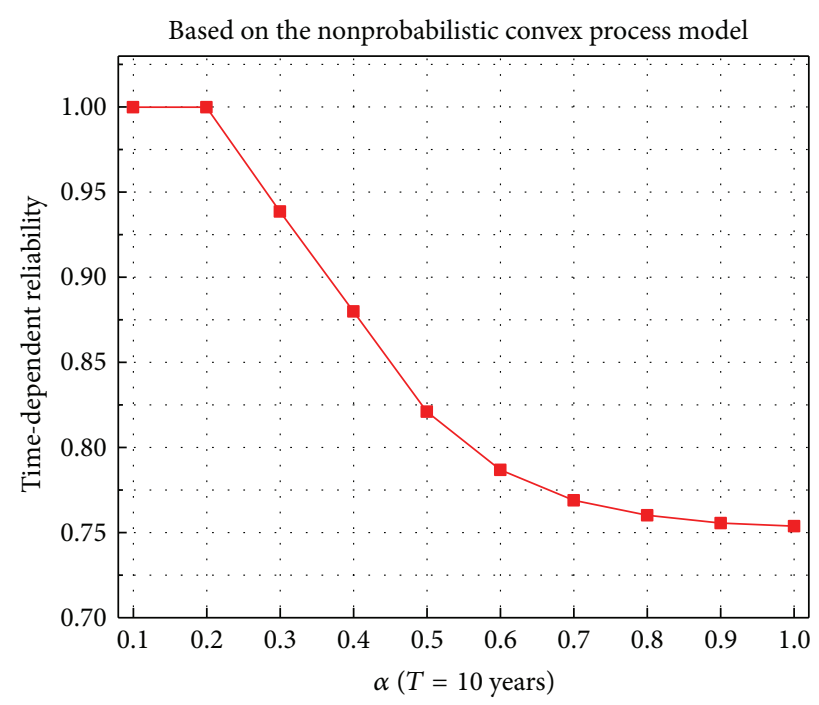

FIGURE 18: Time-dependent reliability results versus $\alpha$.

case of $\alpha=1$ ), and its variation is essentially linear at the beginning and then becomes nonlinear to a certain degree. 
TABLE 7: Time-varying uncertainty characteristics of the marine propeller structure.

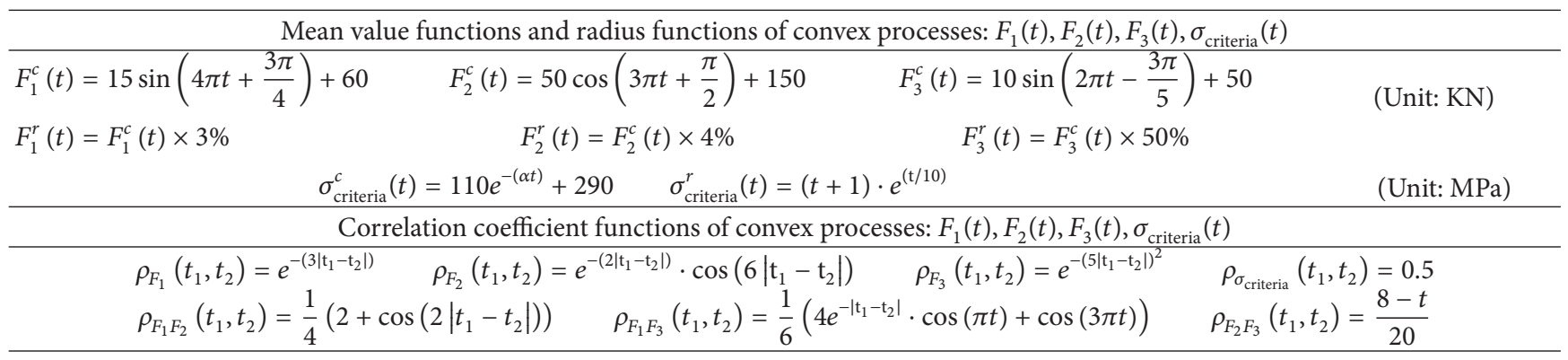

TABLE 8: Reliability results based on the nonprobabilistic convex process model.

\begin{tabular}{ccccccccccc}
\hline$\alpha$ & $\alpha=0.1$ & $\alpha=0.2$ & $\alpha=0.3$ & $\alpha=0.4$ & $\alpha=0.5$ & $\alpha=0.6$ & $\alpha=0.7$ & $\alpha=0.8$ & $\alpha=0.9$ & $\alpha=1.0$ \\
\hline$k=1$ & 1 & 1 & 0.9387 & 0.88 & 0.8211 & 0.7869 & 0.769 & 0.7602 & 0.7556 & 0.7538 \\
\hline
\end{tabular}

6.4. Discussions on the Computational Results. Synthesizing the computational results of the above three numerical examples, the following points can be inherited:

(1) The characteristics of the time-varying uncertain parameters generally exert a great influence on structural safety. On the one hand, the increasing dispersion and decreasing autocorrelation may result in a severe situation of reduced time-dependent reliability (mainly consulted by Section 6.1); on the other hand, the role of cross-correlation between time-varying uncertain parameters makes analysis more complicated (referred by Section 6.2).

(2) Because less assumptions of uncertainty are needed, more conservative reliability results given by present method appear than those from numerical simulations. However, it should be emphasized that the structural reliability is closely related to the uncertain parameters, and hence subjective assumptions may yield unreliable results, especially when dealing with the engineering cases of limited samples.

(3) Directed at simple problems of dynamics, the MonteCarlo simulation method, as an optional way, can evaluate structural reliability by means of sufficient samples and cumulative operations (such as Sections 6.1 and 6.2) but is powerless when tackling mechanical problems, which contain multidimensional uncertainties, cross-correlation, largescale configurations, or complex boundary conditions (as stated in Section 6.3 in this study).

In summary, the numerical examples demonstrate that the time-dependent reliability analysis based on nonprobabilistic convex process model has high efficiency and simultaneously an acceptable analysis precision. More importantly, we can provide a feasible and reasonable way to mathematically evaluate the dynamical safety for complex engineering problems.

\section{Conclusions}

With the rapid technological advance, the reliability analysis considering time-varying effect has attracted more and more concerns and discussions. Currently, most of the approaches for performing time-dependent reliability assessment are always based upon the random process model, where the distributions of time-varying uncertain parameters should be determined by a substantial number of samples, which, however, are not always available or sometimes very costly for practical problems. Thus some assumptions on distribution characteristics have to be made in many dynamical cases when using the probability model. Nevertheless, unjustified assumptions may give rise to misleading results unexpectedly.

In view of the abovementioned facts, this paper describes the time-varying uncertainty with the model of nonprobabilistic convex process. In this convex process model, the uncertain variables at any time are expressed as intervals and the corresponding autocovariance function and autocorrelation coefficient function are established to characterize the relationship between variables at different times. Referred by the definitions in random process theory, the cross-correlation between different two convex processes is also considered. Then, by using the set-theory method and the regularization technique, the time-varying limit-state function is transformed and quantified by a standard convex process model with autocorrelation. Enlightened by the ideas of first-passage method and static reliability analysis, a new nonprobabilistic measurement of time-dependent reliability is proposed, and its analytical expression in linear case is conducted mathematically. Additionally, as a means of verification and comparison, the Monte-Carlo simulation method is also presented and applied into the solution of numerical examples. Analytical results indicate that the present method can be ensured to be more applicable and efficient when estimating structural safety of complex engineering problems.

Indeed, the present time-dependent reliability analysis technique based on the nonprobabilistic convex process model can be regarded as a beneficial supplement to the current reliability theory of random processes. On the basis of the reliability analysis, the proposed safety measurement index can be also applied to the fields of time-varying structural design optimization. 


\section{Conflict of Interests}

The authors declare that there is no conflict of interests regarding the publication of this paper.

\section{Acknowledgments}

The authors would like to thank the National Nature Science Foundation of China (no. 11372025) for the financial supports. Besides, the authors wish to express their many thanks to the reviewers for their useful and constructive comments.

\section{References}

[1] C. Andrieu-Renaud, B. Sudret, and M. Lemaire, "The PHI2 method: a way to compute time-variant reliability," Reliability Engineering and System Safety, vol. 84, no. 1, pp. 75-86, 2004.

[2] H. O. Madsen, S. Krenk, and N. C. Lind, Methods of Structural Safety, Dover, New York, NY, USA, 2006.

[3] M. Lemaire, Structural Reliability, ISTE-Wiley, New York, NY, USA, 2009.

[4] Z. Wang and P. Wang, "A new approach for reliability analysis with time-variant performance characteristics," Reliability Engineering \& System Safety, vol. 115, pp. 70-81, 2013.

[5] A. M. Haofer, "An exact and invariant first-order reliability format," Journal of Engineering Mechanics, vol. 100, pp. 111-121, 1974.

[6] J. F. Zhang and X. P. Du, "A second-order reliability method with first-order efficiency," Journal of Mechanical Design, Transactions of the ASME, vol. 132, no. 10, Article ID 101006, 2010.

[7] L. A. Zadeh, "Fuzzy sets as a basis for a theory of possibility," Fuzzy Sets and Systems, vol. 1, no. 1, pp. 3-28, 1978.

[8] Y. Ben-Haim, "A non-probabilistic concept of reliability," Structural Safety, vol. 14, no. 4, pp. 227-245, 1994.

[9] Y. J. Luo, Z. Kang, and A. Li, "Structural reliability assessment based on probability and convex set mixed model," Computers \& Structures, vol. 87, no. 21-22, pp. 1408-1415, 2009.

[10] C. Jiang, G. Y. Lu, X. Han, and L. X. Liu, "A new reliability analysis method for uncertain structures with random and interval variables," International Journal of Mechanics and Materials in Design, vol. 8, no. 2, pp. 169-182, 2012.

[11] G. Barone and D. M. Frangopol, "Reliability, risk and lifetime distributions as performance indicators for life-cycle maintenance of deteriorating structures," Reliability Engineering and System Safety, vol. 123, pp. 21-37, 2014.

[12] J. Li, J.-B. Chen, and W.-L. Fan, "The equivalent extremevalue event and evaluation of the structural system reliability," Structural Safety, vol. 29, no. 2, pp. 112-131, 2007.

[13] Z. Wang, Z. P. Mourelatos, J. Li, I. Baseski, and A. Singh, “Timedependent reliability of dynamic systems using subset simulation with splitting over a series of correlated time intervals," Journal of Mechanical Design, vol. 136, Article ID 061008, 2014.

[14] M. Mejri, M. Cazuguel, and J. Y. Cognard, "A time-variant reliability approach for ageing marine structures with nonlinear behaviour," Computers \& Structures, vol. 89, no. 19-20, pp. 1743-1753, 2011.

[15] G. Tont, L. Vladareanu, M. S. Munteanu, and D. G. Tont, "Markov approach of adaptive task assignment for robotic system in non-stationary environments," Wseas Transactions on Systems, vol. 9, no. 3, pp. 273-282, 2010.
[16] V. Bayer and C. Bucher, "Importance sampling for first passage problems of nonlinear structures," Probabilistic Engineering Mechanics, vol. 14, no. 1-2, pp. 27-32, 1999.

[17] S. O. Rice, "Mathematical analysis of random noise," The Bell System Technical Journal, vol. 23, pp. 282-332, 1944.

[18] E. H. Vanmarcke, "On the distribution of the first-passage time for normal stationary random processes," Journal of Applied Mechanics, Transactions ASME, vol. 42, no. 1, pp. 215-220, 1975.

[19] P. H. Madsen and S. Krenk, "An integral equation method for the first-passage problem in random vibration," Journal of Applied Mechanics, vol. 51, no. 3, pp. 674-679, 1984.

[20] Z. Hu and X. Du, "Time-dependent reliability analysis with joint upcrossing rates," Structural and Multidisciplinary Optimization, vol. 48, no. 5, pp. 893-907, 2013.

[21] G. Schall, M. H. Faber, and R. Rackwitz, "The ergodicity assumption for sea states in the reliability estimation of offshore structures," Journal of Offshore Mechanics and Arctic Engineering, vol. 113, no. 3, pp. 241-246, 1991.

[22] S. Engelund, R. Rackwitz, and C. Lange, "Approximations of first-passage times for differentiable processes based on higherorder threshold crossings," Probabilistic Engineering Mechanics, vol. 10, no. 1, pp. 53-60, 1995.

[23] H. Streicher and R. Rackwitz, "Time-variant reliability-oriented structural optimization and a renewal model for life-cycle costing," Probabilistic Engineering Mechanics, vol. 19, no. 1, pp. 171-183, 2004.

[24] Q. Li, C. Wang, and B. R. Ellingwood, "Time-dependent reliability of aging structures in the presence of non-stationary loads and degradation," Structural Safety, vol. 52, pp. 132-141, 2015.

[25] Z. P. Mourelatos, M. Majcher, V. Pandey, and I. Baseski, "Time-dependent reliability analysis using the total probability theorem," Journal of Mechanical Design, vol. 137, no. 3, Article ID 031405, 2015.

[26] B. Sudret, "Analytical derivation of the outcrossing rate in time-variant reliability problems," Structure and Infrastructure Engineering, vol. 4, no. 5, pp. 353-362, 2008.

[27] C. Jiang, B. Y. Ni, X. Han, and Y. R. Tao, "Non-probabilistic convex model process: a new method of time-variant uncertainty analysis and its application to structural dynamic reliability problems," Computer Methods in Applied Mechanics and Engineering, vol. 268, pp. 656-676, 2014.

[28] X. Wang, L. Wang, I. Elishakoff, and Z. Qiu, "Probability and convexity concepts are not antagonistic," Acta Mechanica, vol. 219, no. 1-2, pp. 45-64, 2011. 


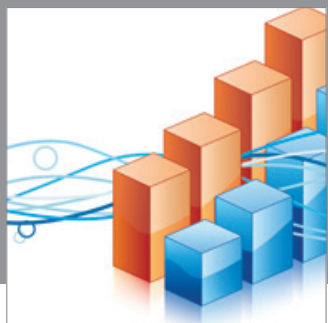

Advances in

Operations Research

mansans

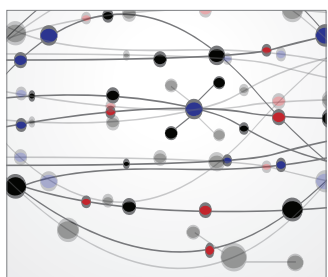

The Scientific World Journal
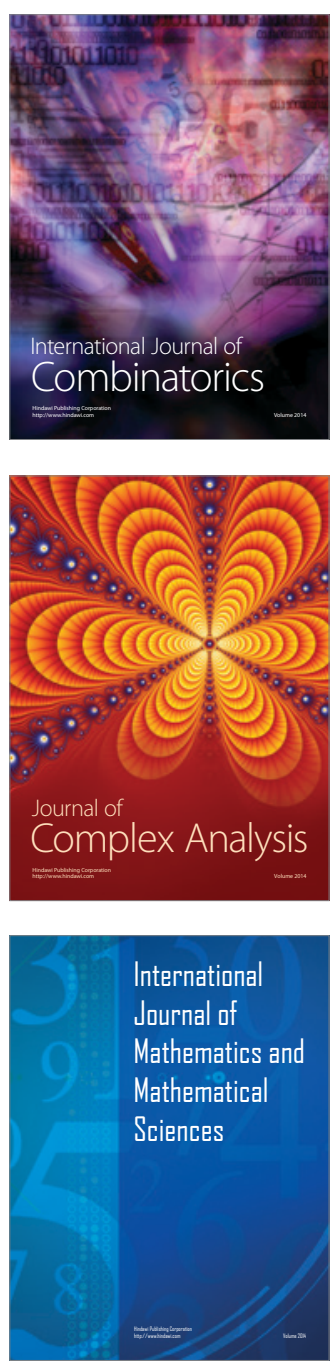
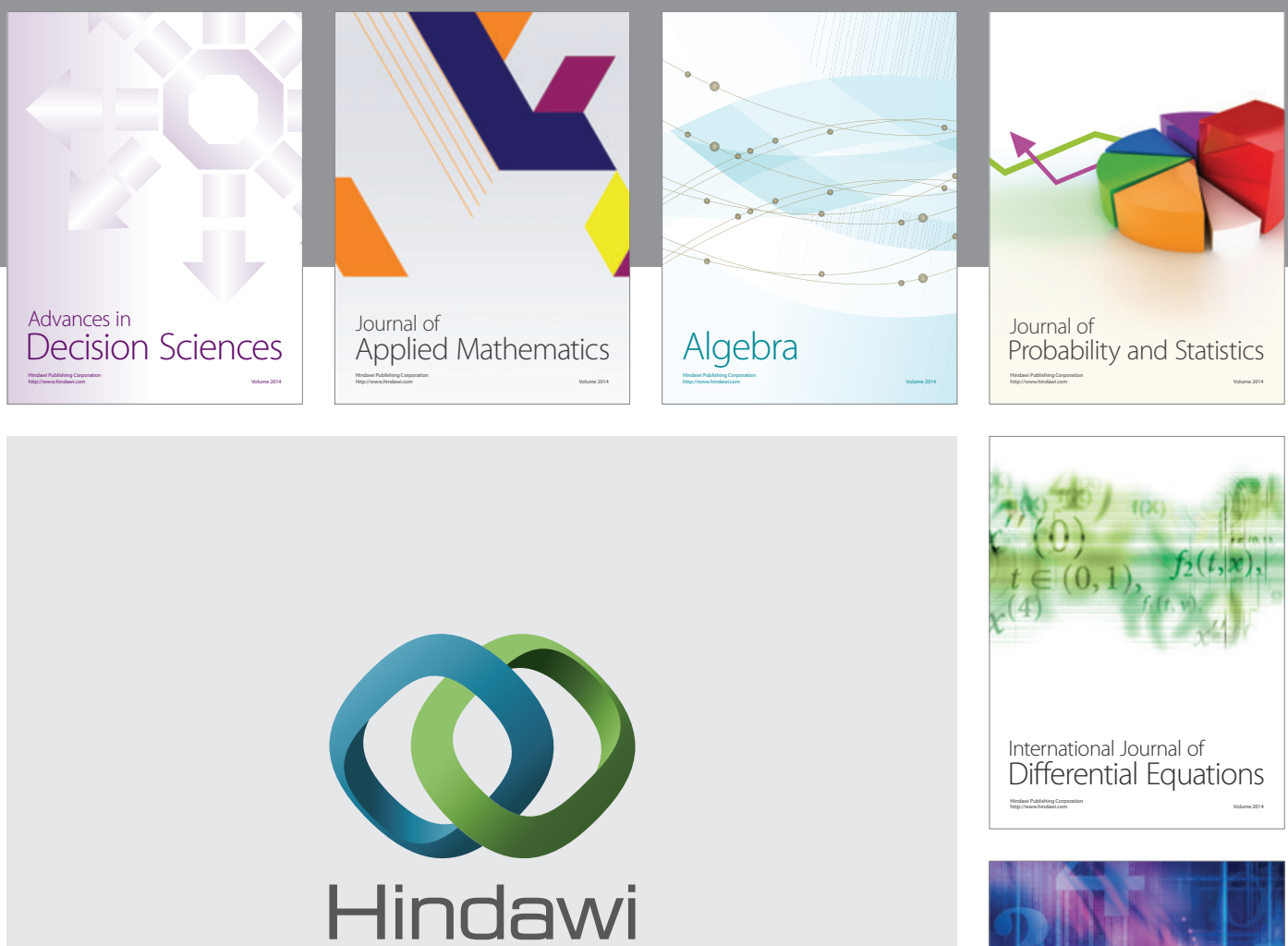

Submit your manuscripts at http://www.hindawi.com
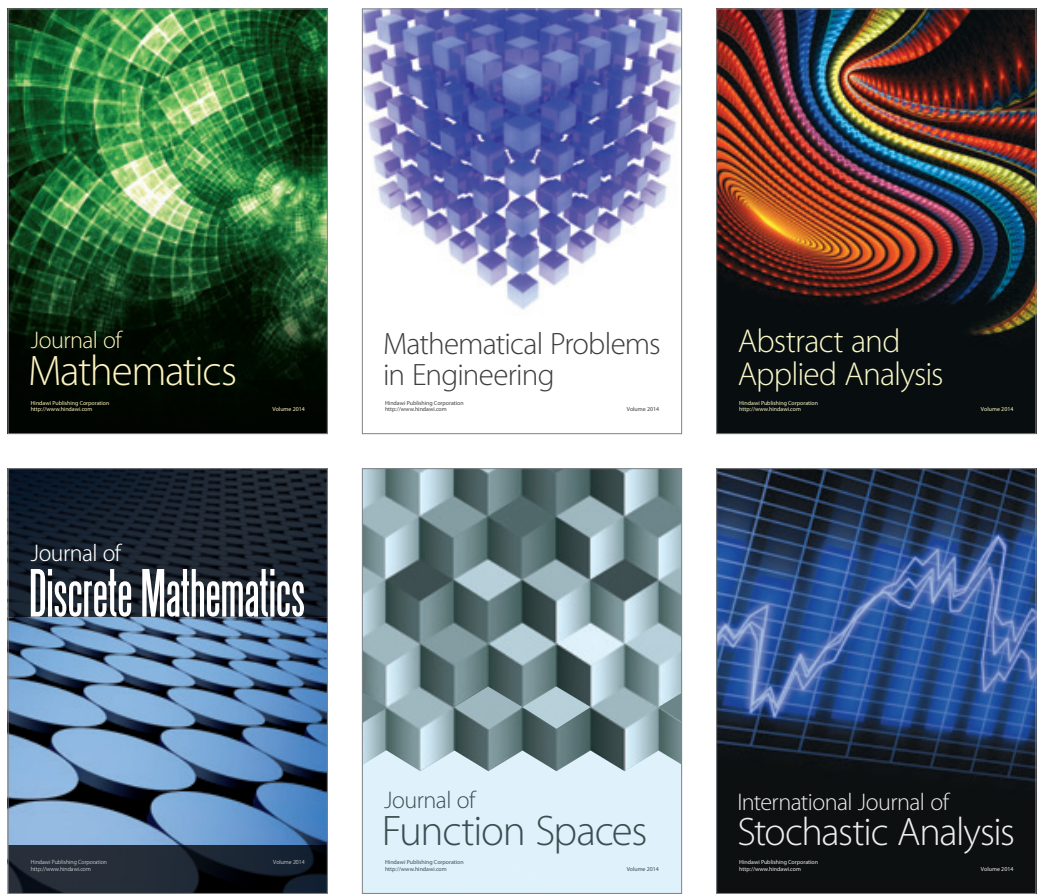

Journal of

Function Spaces

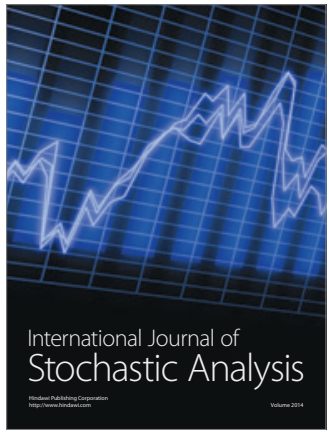

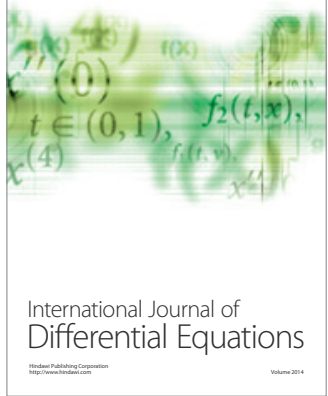
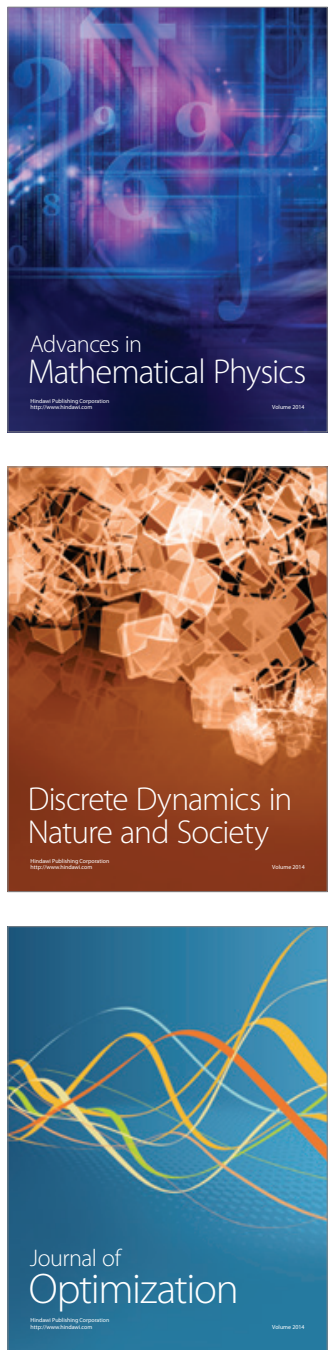